\title{
Low-carbon tourism strategy evaluation and selection using interval-valued intuitionistic fuzzy additive ratio assessment approach based on similarity measures
}

\author{
Arunodaya Raj Mishra $^{1}$ (D) Ayushi Chandel ${ }^{2} \cdot$ Parvaneh Saeidi $^{3}$
}

Received: 11 May 2021 / Accepted: 6 August 2021 / Published online: 3 September 2021

(c) The Author(s), under exclusive licence to Springer Nature B.V. 2021

\begin{abstract}
Recently, the assessment and selection of most suitable low-carbon tourism strategy has gained an extensive consideration from sustainable perspectives. Owing to participation of multiple qualitative and quantitative attributes, the low-carbon tourism strategy (LCTS) selection process can be considered as multi-criteria decision-making (MCDM) problem. As uncertainty is usually occurred in LCTSs evaluation, the theory of interval-valued intuitionistic fuzzy sets (IVIFSs) has been established as more flexible and efficient tool to model the uncertain decision-making problems. The idea of the present study is to develop an extended method using additive ratio assessment (ARAS) framework and similarity measures in a way to find an effective solution to the decision-making problems using IVIFSs. The bases of the proposed method are the IVIFSs operators, some modifications in the traditional ARAS framework and a calculation procedure of the weights of the criteria. To calculate criterion weight, new similarity measures for IVIFSs are developed aiming at the achievement of more realistic weights. Also, a comparison is demonstrated to the currently used similarity measures in order to show the efficiency of the developed approach. To confirm that the developed IVIF-ARAS approach can be successfully employed to practical decision-making problems, a case study of LCTS selection problem is considered. The final results from the developed approach and the extant models are compared for the validation of the proposed approach in this study.
\end{abstract}

Keywords Interval-valued intuitionistic fuzzy sets · Similarity measure · MCDM · ARAS · Low-carbon tourism

Arunodaya Raj Mishra

arunodaya87@outlook.com

Ayushi Chandel

ashuchande1.95@gmail.com

1 Department of Mathematics, Government College Jaitwara, Satna, Madhya Pradesh 485221, India

2 Department of Computer Science \& Engineering, ITM University, Gwalior, Madhya Pradesh 474001, India

3 Facultad de Ciencias Adminsitrativas y Económicas, Universidad Tecnológica Indoamérica, Quito, Ecuador 


\section{Introduction}

During these days, a series of new ideas and policies have emerged, namely "carbon footprint" (He et al., 2020), "low-carbon economy (LCE)" (Bonsu, 2020), "low-carbon technology" (Shi et al., 2020), "low-carbon development" (Chen et al., 2020), "low-carbon lifestyle" (Cheng et al., 2020), "low-carbon supply chain (LCSC)" (Shaharudin et al., 2019), "low-carbon society (LCS)" (Berger et al., 2020), "low-carbon city" (Harris et al., 2020) and "low-carbon world (Cojoianu et al., 2020)". In the contextual of global warming, the "LCE" based on low energy consumption and pollution has become a global hot spot (He $\& \mathrm{Tu}, 2020)$. Developed countries in Europe and the USA vigorously promote the "lowcarbon revolution" (Wen \& Jing, 2011) with energy efficiency and low emissions as the core, focus on the progress of "low-carbon technology", and make major adjustments to industries, energy, technology, trade and other policies in order to seize the opportunities and occupy the industrial commanding heights.

With the increasing global warming and environmental concerns, a wave of LCE action has received a great attention. Low-carbon tourism, as an extension of LCE, is a significant way to achieve the sustainable growth of the tourism sector and a development of future economic growth (Zhang et al., 2020). The low-carbon method mentions the lessening of $\mathrm{CO}_{2}$ and other GHG emissions through policy management, technological innovation, and alterations in individual life activities in the combined efforts of the government, organizations, and individuals to make a way of economic growth of low-carbon emission. The low-carbon mode can not only encounter the requirements of economic development, but also guard the environment (Mu et al., 2011; Su, 2019).

The low-carbon tourism (LCT) enterprise has immense social value and economic importance (He \& Wang, 2014). In 2008, Gössling et al. (2012) presented the concern of LCT and asserted that it is sustainable. Also, United Nations World Tourism Organization (UNWTO) (2008) produced data on universal Tourism Carbon Emissions (TCEs), which benefits the academic circles and the tourism sector to achieve intuitive perception of the problem of TCEs. Given the contextual pervasiveness of a low-carbon idea, several authors have focused their attention on this phenomenon and presented considerable researches (Sect. 2.1). Though there is no universal definition of LCT, the key prominence is that energy consumption and $\mathrm{CO}_{2}$ emissions initiated by the actions, goods and services of tourism are minimized (Chiesa \& Gautam, 2009; Filimonau et al., 2011). In recent years, a few of previous studies highlighted the important role of low-carbon tourism in the literature review (Lee et al., 2018; Puška et al., 2019; Thongdejsri \& Nitivattananon, 2019; Zhang, 2017; Zhang \& Zhang, 2020). He et al. (2021) gave a framework for conceptualizing the emerging sustainable community-based tourism using a SWARA (step-wise weight assessment ratio analysis)-MULTIMOORA (multi-objective optimization based on ratio analysis with full multiplicative form) methodology.

However, the selection of desirable LCT strategy can be regarded as multi-criteria decision-making (MCDM) problem due to occurrence of several tangible and intangible attributes (Zhang, 2017). Recently, several MCDM methods have been effectively implemented for solving various practical decision-making problems. Nonetheless, the majority of MCDM methods work with crisp numbers, which might be insufficient when working on real-life problems. In real-life applications, the selection of the optimal options often relies on fuzzy and uncertain information. Thus, the majority of MCDM approaches are extended in a way to be applicable to fuzzy environments. In some instances, fuzzy numbers may be still inadequate because of the natural vagueness 
of subjective decisions made by decision experts (DEs) or the vagueness of the complicated socio-economic situations. As decision-making problems turn out to be more complex, recognizing a distinctive alternative becomes more challenging for DEs. In this situation, the fuzzy doctrine is applied for evaluating the best alternative due to its resemblance to human analysis. In addition, we observe that information may also suffer from some limitations, which results in insufficiency and lack of accuracy. Such flaws of traditional FSs or crisp sets can be dealt by employing the IFSs, which can capture DEs' judgments (Büyüközkan \& Göçer, 2017).

When DEs make a decision to assess and choose an appropriate option, this generally recommends a complicated problem concerning numerous criteria. To manage it effectively, additive ratio assessment (ARAS) method is an appropriate approach to adopt. ARAS mainly relies upon the inspiration that the philosophy of complex domains with contradictory attributes can be explained by applying easy relative associations as pioneered by Zavadskas and Turskis (2010). The outcome of the paper arrives from its proposal of a new MCDM method incorporating the interval-valued intuitionistic fuzzy sets (IVIFSs)-similarity measures and conventional ARAS approach. In the extended ARAS method, the performance evaluations of alternatives are articulated in terms of IVIFSs, whereas similarity measure is applied for criteria evaluation in a decision-making setting, where DE expresses their individual performance evaluation with IVIFNs. The developed method was found more flexible in determining group performance evaluations over the others. Combining the similarity measure and ARAS approach on IVIFSs facilitates DEs to employ the advantages of the similarity measure, which are attained because of its capability in estimating problems linearly and ARAS approach offered by its capability in assessing experts' preferences efficiently. This association on IVIFSs offers a simple and more effective approach to solving complicated decision-making problems. It also offers a useful, sensible and logical outcome in decision-making procedure on IVIFSs considerable strength in relating the uncertain and fuzzy atmosphere as a benefit on the fuzzy and intuitionistic fuzzy outlines of similarity measure and ARAS method. Then, the proposed method is implemented on low-carbon tourism strategy (LCTS) selection problem. This combination has a contribution to existing body of knowledge through pioneering an incorporated framework and making use of a real-life problem to develop the LCTS process. The main benefit of intuitionistic fuzzy sets (IFSs) compared to the crisp or a conventional fuzzy doctrine is that IFSs distinguish the negative and the positive suggestion for membership and non-membership options (Büyüközkan \& Gocer, 2017). The IVIFSs doctrine was pioneered by Atanassov and Gargov (1989) for handling the uncertainty in the information and fuzziness in DE's opinions in realistic decision-making concerns. The prime outcome of IVIFSs is that both the belongingness membership grade (BG) and the non-belongingness grade (NG) of an element to IVIFSs are considered and measured on interval values instead of exact numbers. As a result, there is a significant need to explore more effectual and suitable mathematical methods by employing the IVIFSs consecutively to better tackle low-carbon tourism strategy selection problems with higher levels of uncertainty and ambiguity. On the contrary, IVIFNs offer an opportunity for providing a model of a higher sufficiency for evaluating the complicated real-life problems. IVIF-ARAS is known as a discrete method whose usefulness is established in the present research.

However, according to the above discussions, in this study, an attempt has been carried out to develop a comprehensive framework to evaluate and selection the LCTSs in Indian tourism areas. Therefore, the main contributions of this study are provided as follows:

Firstly, a new comprehensive framework to evaluate and selection of LCTSs in the Indian tourism areas has been developed. 
Secondly, an extended ARAS approach under IVIF environment is introduced to evaluate and selection of LCTSs in the Indian tourism areas.

Thirdly, a survey and literature review related to similarity measure for IVIFSs is conducted. Novel similarity measures for IVIFSs are developed to obtain more realistic weights. Also, comparative discussion is made with extant method to show superiority of developed ones.

Fourthly, the result of IVIF-ARAS is compared with the IVIF-TOPSIS, IVIF-COPRAS, ANP-GCI and IVIF-MABAC methods to illustrate the proficiency and consistency of proposed framework.

The remaining study is organized as Sect. 2 that confers the literature review related to low-carbon tourism strategy evaluation and ARAS method. Section 3 offers the basic concepts of IVIFSs, proposes a novel similarity measure and develops an extended IVIFARAS methodology. Section 4 demonstrates a case study of LCTS selection which displays the practicality and usefulness of developed model. Lastly, Sect. 5 concludes the study and recommends for future study.

\section{Literature review}

\subsection{An overview on low-carbon tourism}

Tourism is one of the fastest developing industries in the globe. As travel demands will only continue to grow, both tourism operators and tourists will undoubtedly become the producers of today's global greenhouse effect. They have the accountability and commitment to sponsor and practice low-carbon green tourism. The root cause of such events is the global warming mainly caused by industrial development, together with the increase in the total emissions of individual carbon dioxide in transportation and various consumption behaviors in the tourism industry (Yu \& Tsung-Lin, 2020). By comparing with issues about an LCE, LCT only acknowledged attention much later. However, in the commencement of this century, tourism or research into the association with the tourism development and $\mathrm{CO}_{2}$ emissions has gained growing concentration, and the idea of "LCT" was not put forward until 2008. Gössling et al. (2012) gave the concern of "moving toward low-carbon tourism: new opportunities for destinations and tour operators." Gössling et al. (2015) authorize the predominance of transportation in tourism carbon emissions. Durbarry and Seetanah (2015) explore the association between tourism carbon emissions (TCEs) and climate change. Tourism operator priorities and inspirations for carbon offset choices by ecologically licensed tourism enterprises, the significance of communications in employing carbon label structures, as well as the carbon literacy of hotel staff and tourists' understanding of a LCT product were inspected by Zeppel \& Beaumont (2013), Gössling and Buckley (2016), Teng et al. (2014), McKercher et al. (2010) and Juvan and Dolnicar (2014), respectively. Correspondingly, Horng et al. (2014) focused on the significance of low-carbon edification in the determination to attain TCEs reductions. McLennan et al. (2014) criticized the detail that the uptake of carbon offsetting projects has been apparently quite low in the tourism industry, notwithstanding the extensive variety of these structures.

Despite the sustainable efforts in LCT, as opposed to the assessment of an LCE (Huang \& Mauerhofer, 2016; Zhou et al., 2015), to our information, some researchers have developed to the assessment of LCT, particularly regarding the meso/macro-tourism destinations. For instance, Cheng et al. (2013) discussed an assessment index scheme containing 
27 diverse assessment indices concerned with the environment, management and attitudes to LCT attractions. They applied a wetland as a case study, while Luo et al. (2014) gave a structure dynamics scheme of a de-carbonated tourist attraction with 12 level variables. Furthermore, preceding relevant studies on assessment indices of LCT development have been accompanied, containing: the association and growth tendencies between revenues, energy consumption, garbage emission and carbon intensity, as explored by (Xu et al., 2011). Taiwan's LCT development suitability assessment indicators were recognized by (Cho et al., 2016). They created a thorough assessment index scheme containing of 53 indicators, which discusses transportation, travel agencies, hotel accommodation, destinations, local communities and food service.

Though, the outcomes of discussed researches are always tough to implement to real policy-making in LCT development as (a) various indicators may diminish the efficiency of management in maximum cases, (b) the linear assessment does not resemble well with indicators which have multifaceted interdependence between them and (c) some indicators, namely resident population and information scheme construction are beyond the LCT structure, notwithstanding their association with LCT. That means, the index structure is too generalized. Thus, this paper aims to present a new decision-making method which can be applied to assess LCTSs and offer some countermeasures to propose LCT. The key outcome of this study is to determine the LCTSs and set up a widespread and effective regional assessment indicator structure of LCT.

\subsection{A review of fuzzy decision-making approach}

MCDM approaches are adopted for the aim of selecting the optimal alternative from the available alternatives set over various conflicting criteria. The criterion information is determined to achieve the optimum outcome in MCDM approaches. Recently, in decision-making settings, FSs and their extensions have obtained more attention from the scholars, which was mainly due to the increase of complexity and widespread changes of today's environment. As a result, lots of MCDM methods including elimination and choice expressing reality (ELECTRE) (Amirghodsi et al., 2020; Kilic et al., 2020; Mishra et al., 2020d), TOPSIS (Rani et al., 2020a, 2020c), TODIM (an acronym in Portuguese for interactive MCDM) (Mishra et al., 2020a, 2020b, 2020c, 2020d; Wang et al., 2020), VIKOR (Rafieyan et al., 2020; Rani et al., 2019), PROMETHEE (Preference ranking organization method for enrichment of evaluations) (Greco et al., 2021; Makan \& Fadili, 2020), WASPAS (weighted aggregated sum product assessment), complex proportional assessment (COPRAS) (Mishra et al., 2019, 2020c; Rani et al., 2020b), ARAS (Heidary Dahooie et al., 2019; Zavadskas \& Turskis, 2010), and others were generalized in an uncertain decisionmaking context with varied weight-determination approaches.

The IVIFSs doctrine was pioneered by Atanassov and Gargov (1989) for handling the uncertainty in the information and fuzziness in DE's opinions in real decision-making problems. Various author(s) have utilized IVIFSs to construct MCDM approaches for solving real-life problems under uncertain situations (Mishra et al., 2020a, 2020b; Wang \& Wan, 2020; Chen et al., 2021). First, Xu (2007b) proposed various aggregation operators for IVIFSs and presented an application of the intuitionistic fuzzy weighted geometric average (IFWGA) and IVIF-weighted arithmetic average (IVIFWAA) operators to MCDM problems by utilizing the score value and accuracy value of IVIFNs. Bai (2013) introduced an enhanced version of score function to efficiently rank the order of the IVIFNs and 
developed score function based IVIF-TOPSIS approach to evaluate MCDM problem with completely unknown weight information.

Mishra and Rani (2018b) studied an extended WASPAS model using entropy, divergence and similarity measures for evaluating reservoir flood control management policy. Narayanamoorthy et al. (2019) gave a model using fuzzy VIKOR under interval-valued intuitionistic hesitant fuzzy entropy for selection of industrial robots. Yeni and Özçelik (2019) proposed a new method using combinative distance-based assessment (CODAS) approach under IVIFSs for handling group decision-making problem. Abdullah et al. (2019) presented a novel decision-making method based on modification of DEMATEL approach under IVIFS environment and Choquet integral for solving sustainable solid waste management problems. Mishra et al. (2020b) suggested a novel TODIM method to assess and select desirable vehicle insurance company under IVIFSs environment.

Alternatively, several researchers have made effective use of ARAS and fuzzy ARAS in various disciplines, e.g., sustainable development, economics, engineering, and construction (see Table 1). The assessment provided in Table 1 implies the combined similarity measure and ARAS for IVIFSs on decision making is unique and has not been implemented before.

\section{Research method and materials}

\subsection{Preliminaries}

Here, we present some fundamental notions of IVIFSs.

Definition 1 (Atanassov \& Gargov, 1989). Let $U=\left\{u_{1}, u_{2}, \ldots, u_{n}\right\}$ be a common worldwide set. The IVIFS $S$ in $U$ is an object based on the mathematical view as follows:

$$
S=\left\{\left\langle u_{i}, \mu_{S}\left(u_{i}\right), v_{S}\left(u_{i}\right)\right\rangle: u_{i} \in U\right\},
$$

where $\mu_{S}, v_{S}: U \rightarrow[0,1]$ hold $\sup \left(\mu_{S}\left(u_{i}\right)\right)+\sup \left(v_{S}\left(u_{i}\right)\right) \leq 1$. The intervals $\mu_{S}\left(u_{i}\right)$ and $v_{S}\left(u_{i}\right)$ stand for BG and $\mathrm{NG}$ of an object $u_{i}$ in the set $U$, respectively.

For simplicity, suppose $\mu_{S}\left(u_{i}\right)=\left[\mu_{S}^{-}\left(u_{i}\right), \mu_{S}^{+}\left(u_{i}\right)\right]$ and $v_{S}\left(u_{i}\right)=\left[v_{S}^{-}\left(u_{i}\right), v_{S}^{+}\left(u_{i}\right)\right]$, then

$$
S=\left\{\left\langle u_{i},\left[\mu_{S}^{-}\left(u_{i}\right), \mu_{S}^{+}\left(u_{i}\right)\right],\left[\nu_{S}^{-}\left(u_{i}\right), v_{S}^{+}\left(u_{i}\right)\right]\right\rangle: u_{i} \in U\right\},
$$

where $\quad 0 \leq \mu_{S}^{+}\left(u_{i}\right)+v_{S}^{+}\left(u_{i}\right) \leq 1, \mu_{S}^{-}\left(u_{i}\right) \geq 0 \quad$ and $\quad v_{S}^{-}\left(u_{i}\right) \geq 0$. The interval $\pi_{S}\left(u_{i}\right)=\left[\pi_{S}^{-}\left(u_{i}\right), \pi_{S}^{+}\left(u_{i}\right)=\left[1-\mu_{s}^{+}\left(u_{i}\right)-v_{S}^{+}\left(u_{i}\right), 1-\mu_{S}^{-}\left(u_{i}\right)-v_{S}^{-}\left(u_{i}\right)\right]\right.$ signifies the hesitancy grade of $u_{i}$ to $S$.

According to Definition 1, an IVIFS is considered by interval-valued BG and NG based on ordered pair. For a given $u_{i} \in U$, the pair $\left(\left[\mu_{S}^{-}\left(u_{i}\right), \mu_{S}^{+}\left(u_{i}\right)\right],\left[v_{S}^{-}\left(u_{i}\right), v_{S}^{+}\left(u_{i}\right)\right]\right)$ is called an IVIFN (Xu, 2007b). To make it easy, an IVIFN is made typically simpler as $P=([\alpha, \beta],[\gamma, \delta])$, where $[\alpha, \beta] \subset[0,1],[\gamma, \delta] \subset[0,1]$ and $\beta+\delta \leq 1$.

Dymova and Sevastjanov (2016) made an analysis on Definition 1, which was originally offered by Atanassov and Gargov (1989). 


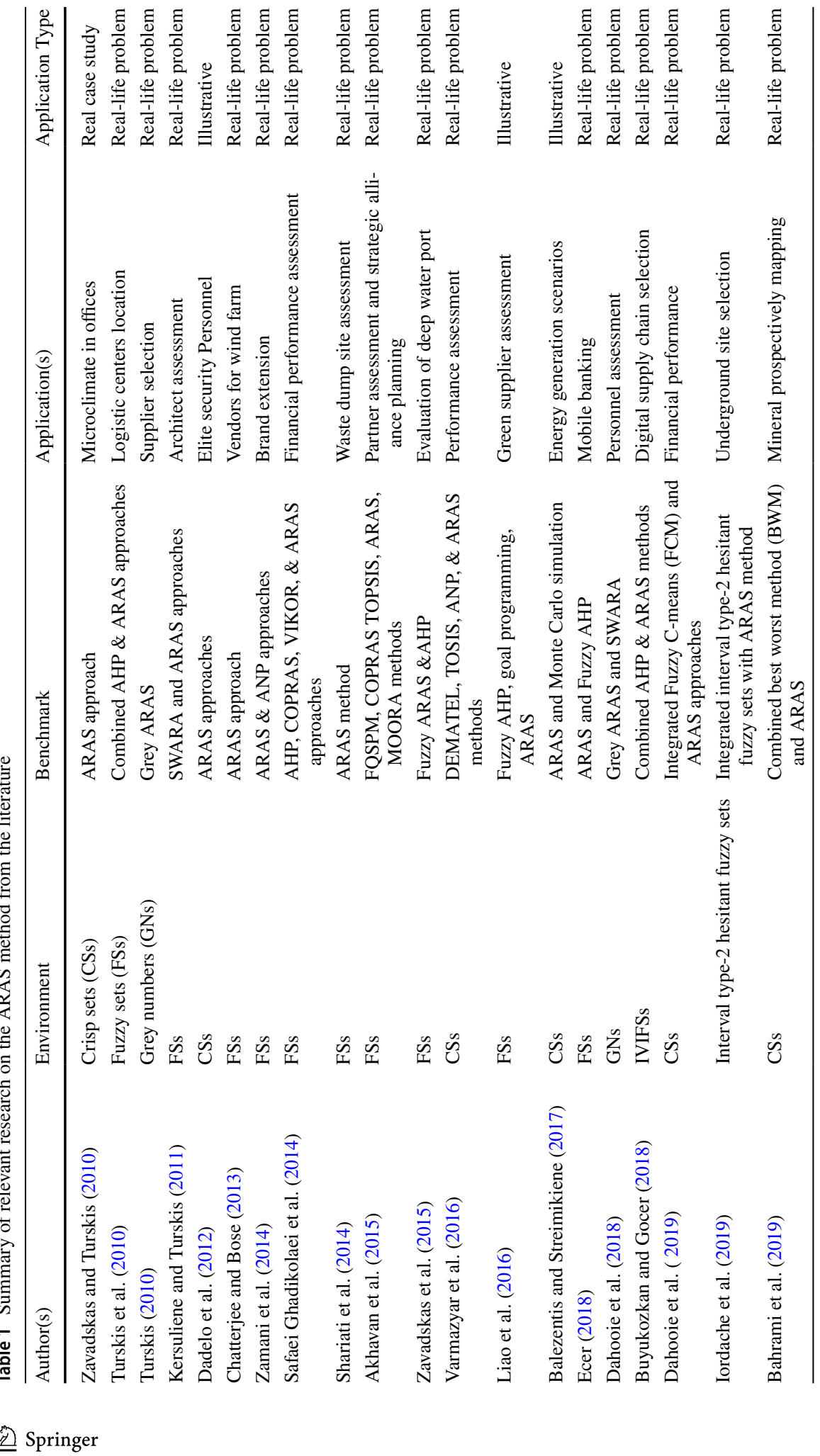




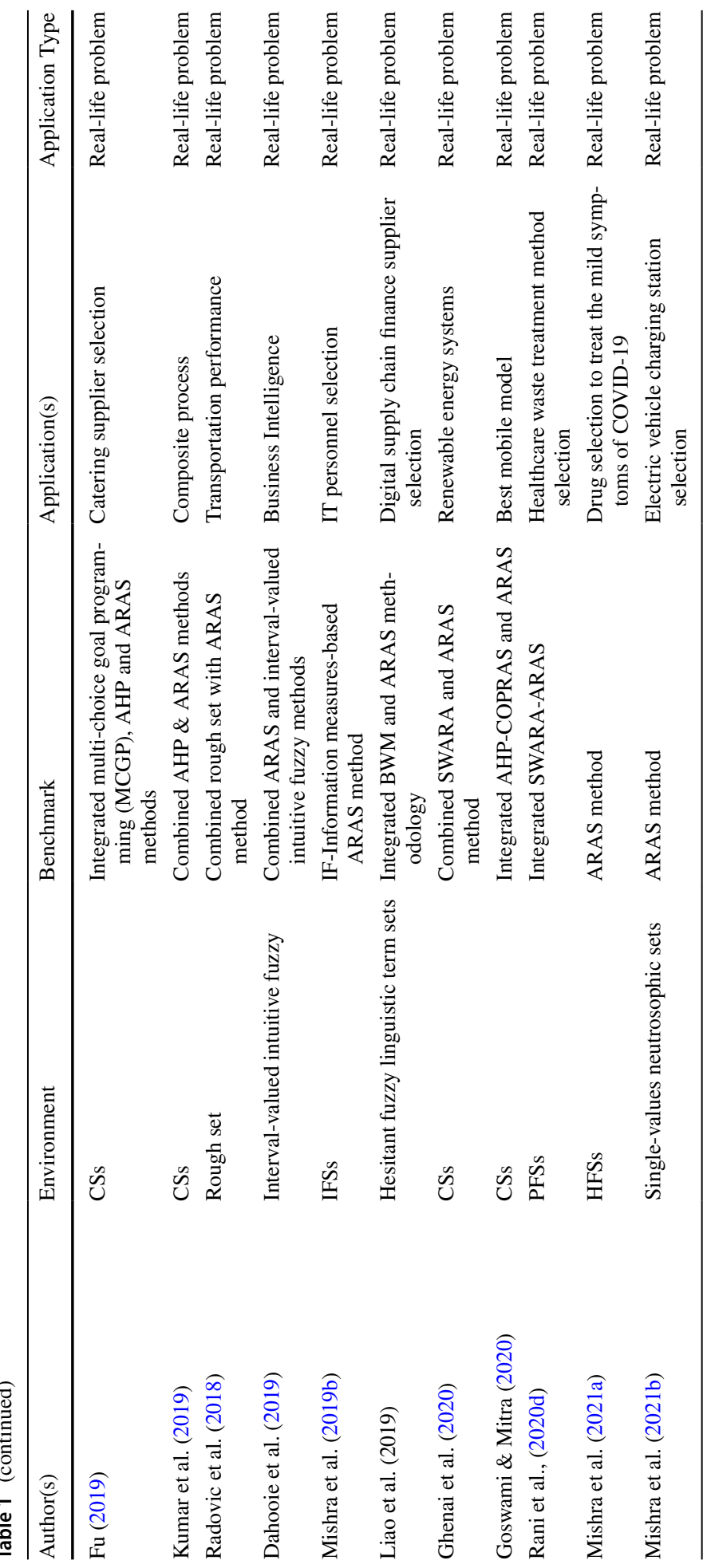


Definition 2 (Dymova \& Sevastjanov, 2016). An IVIFS $S$ in $U$ is an element based on mathematical set:

$$
S=\left\{\left\langle u_{i}, \mu_{S}\left(u_{i}\right), v_{S}\left(u_{i}\right)\right\rangle: u_{i} \in U\right\},
$$

where $\mu_{S}, v_{S}: U \rightarrow[0,1]$ satisfies the constraint $\sup \left(\mu_{S}\left(u_{i}\right)\right)+\inf \left(v_{S}\left(u_{i}\right)\right) \leq 1$ and $\inf \left(\mu_{S}\left(u_{i}\right)\right)+\sup \left(v_{S}\left(u_{i}\right)\right) \leq 1$. The intervals $\mu_{S}\left(u_{i}\right)$ and $v_{S}\left(u_{i}\right)$ signify the BG and NG of an object $u_{i}$ in the set $U$, respectively.

Definition 3 Atanassov and Gargov (1989). Assume $S, T \in \operatorname{IVIFSs}(U)$, then, some operations could be explained as follows:

(a) $S \subseteq T$ iff $\mu_{S}^{-}\left(u_{i}\right) \leq \mu_{T}^{-}\left(u_{i}\right), \mu_{S}^{+}\left(u_{i}\right) \leq \mu_{T}^{+}\left(u_{i}\right), v_{S}^{-}\left(u_{i}\right) \geq v_{T}^{-}\left(u_{i}\right)$ and $v_{S}^{+}\left(u_{i}\right) \geq v_{T}^{+}\left(u_{i}\right)$ for each $u_{i} \in U$;

(b) $S=T$ iff $S \subseteq T$ and $S \supseteq T$;

(c) $S^{c}=\left\{\left\langle u_{i},\left[v_{S}^{-}\left(u_{i}\right), v_{S}^{+}\left(u_{i}\right)\right],\left[\mu_{S}^{-}\left(u_{i}\right), \mu_{S}^{+}\left(u_{i}\right)\right]\right\rangle: u_{i} \in U\right\}$;

(d) $S \cup T=\left\{\left\langle\begin{array}{c}u_{i},\left[\mu_{S}^{-}\left(u_{i}\right) \vee \mu_{T}^{-}\left(u_{i}\right), \mu_{S}^{+}\left(u_{i}\right) \vee \mu_{T}^{+}\left(u_{i}\right)\right], \\ {\left[v_{S}^{-}\left(u_{i}\right) \wedge v_{T}^{-}\left(u_{i}\right), v_{S}^{+}\left(u_{i}\right) \wedge v_{T}^{+}\left(u_{i}\right)\right]}\end{array}\right\rangle: u_{i} \in U\right\}$;

(e) $S \cap T=\left\{\left\langle\begin{array}{c}u_{i},\left[\mu_{S}^{-}\left(u_{i}\right) \wedge \mu_{T}^{-}\left(u_{i}\right), \mu_{S}^{+}\left(u_{i}\right) \wedge \mu_{T}^{+}\left(u_{i}\right)\right], \\ {\left[v_{S}^{-}\left(u_{i}\right) \vee v_{T}^{-}\left(u_{i}\right), v_{S}^{+}\left(u_{i}\right) \vee v_{T}^{+}\left(u_{i}\right)\right]}\end{array}\right\rangle: u_{i} \in U\right\}$.

Definition 4 (Xu, 2007b). Consider $S=\langle[\alpha, \beta],[\gamma, \delta]\rangle$ as an IVIFN. Then,

$$
\mathbb{S}(S)=\frac{1}{2}(\alpha+\beta-\gamma-\delta), \hbar(S)=\frac{1}{2}(\alpha+\beta+\gamma+\delta),
$$

are called score and accuracy values of $S$. Also, $\mathbb{S}(S) \in[-1,1]$ and $\hbar(S) \in[0,1]$.

Since $\mathbb{S}(S) \in[-1,1]$, then $\mathrm{Xu}$ et al. (2015) normalized the score value as follows:

Definition 5 (Xu et al., 2015): Let $S=\langle[\alpha, \beta],[\gamma, \delta]\rangle$ be an IVIFN. Then,

$$
\mathbb{S}^{*}(S)=\frac{1}{2}(\mathbb{S}(S)+1), \hbar^{\circ}(S)=1-\hbar(S),
$$

are known as the improved score and uncertainty functions, respectively. It is clearly observable that $\mathbb{S}^{*}(S) \in[0,1]$ and $\hbar^{\circ}(S) \in[0,1]$.

Definition $6(\mathrm{Xu}, 2007 \mathrm{~b})$ Let $S=\langle[\alpha, \beta],[\gamma, \delta]\rangle$ be an IVIFN and $\xi \in \mathbb{R}$ be an arbitrary real number, then

$$
\xi S=\left\langle\left[1-(1-\alpha)^{\xi}, 1-(1-\beta)^{\xi}\right],\left[\gamma^{\xi}, \delta^{\xi}\right]\right\rangle .
$$

Based on Eq. (1), the definition offered by Xu (2007b) is used as follows:

Let $S=\left\{S_{1}, S_{2}, \ldots, S_{t}\right\}$ be the set of ' $t$ ' IVIFNs such that $S_{k}=\left\langle\left[\alpha_{k}, \beta_{k}\right]\right.$, $\left.\left[\gamma_{k}, \delta_{k}\right]\right\rangle, k=1(1) t$. Then, the IVIF-weighted averaging (IVIFWA) and IVIF-weighted geometric operator (IVIFWG) are given by 


$$
\begin{aligned}
& \bigoplus_{k=1}^{t} \xi_{k} S_{k}=\left\langle\left[1-\prod_{k=1}^{t}\left(1-\alpha_{k}\right)^{\xi_{k}}, 1-\prod_{k=1}^{t}\left(1-\beta_{k}\right)^{\xi_{k}}\right],\left[\prod_{k=1}^{t}\left(\gamma_{k}\right)^{\xi_{k}}, \prod_{k=1}^{t}\left(\delta_{k}\right)^{\xi_{k}}\right]\right\rangle . \\
& \bigotimes_{k=1}^{t} \xi_{k} S_{k}=\left\langle\left[\prod_{k=1}^{t}\left(\alpha_{k}\right)^{\xi_{k}}, \prod_{k=1}^{t}\left(\beta_{k}\right)^{\xi_{k}}\right],\left[1-\prod_{k=1}^{t}\left(1-\gamma_{k}\right)^{\xi_{k}}, 1-\prod_{k=1}^{t}\left(1-\delta_{k}\right)^{\xi_{k}}\right]\right\rangle .
\end{aligned}
$$

Definition 7 (Xu \& Chen, 2008). A function $\operatorname{Sim}: \operatorname{IVIFS}(U) \times \operatorname{IVIFS}(U) \rightarrow[0,1]$ is said to be similarity measure on $\operatorname{IVIFS}(U)$, if it fulfills the given requirements:

(S2) $\operatorname{Sim}(R, S)=1 \Leftrightarrow R=S$;

(S3) $\operatorname{Sim}(R, S)=\operatorname{Sim}(S, R)$;

(S4) For all $R, S, C \in I V I F S s(U)$, if $R \subseteq S \subseteq T$, then $\operatorname{Sim}(R, C) \leq \operatorname{Sim}(R, S)$ and $\operatorname{Sim}(R, C) \leq \operatorname{Sim}(S, C)$.

\subsection{Interval-valued intuitionistic fuzzy similarity measures}

Here, we first review similarity measures (SMs) for IVIFSs. Corresponding to the advantages and practicality of SMs for IFSs, we present SMs for IVIFSs to propose weight determining approach. Then, the results of the IVIF-similarity measures are presented with mathematical evidence and descriptive examples.

\subsubsection{Review of the similarity measures for IVIFSs}

The idea SM is one of the topics with the highest level of attractiveness in IFSs doctrine. It describes the degree of homogeneity between the two IFSs. A SM is a powerful method for decision making (Mishra, 2016; Mishra \& Rani, 2017; Sahu et al., 2021), pattern recognition (Meng \& Chen, 2016) and image processing (Balasubramaniam \& Ananthi, 2014); it has received more consideration in current decades. Dengfeng and Chuntian (2002) first introduced a SM for IFSs. Subsequently, Xu \& Chen (2008) developed a sequence of similarity and distance measures, which are different combinations and extensions of the various distance measures for IFSs and IVIFSs. There are various types of SMs, e.g., entropy measures, similarity persuaded by cosine similarity and interval comparison (Song \& Wang, 2017; Song et al., 2019). In real-life applications, in many cases, information is not available sufficiently, and this is difficult to recognize the precise numbers for the BG and NG. Nonetheless, the decision information may be presented by IVIFSs. Xu (2007a) extended several SMs formulae of IFSs to IVIFSs using IVIF-distance measures. Hu and Li (2013) proposed a system to develop SMs based on entropy measures for IVIFSs. Ye (2013) and Ye (2012) developed the SMs for IVIFSs based on Dice SM and IF-cosine SM (angular coefficient). Meng and Chen (2016) presented new entropy for IVIFSs and a method to develop a SM for IVIFSs. Pekala and Balicki (2017) presented inclusion measure and SM on IVIFSs connected with lexicographical order and discussed various elegant properties. Rani et al. (2018) developed entropy and similarity measures for IVIFSs based on exponential and discussed VIKOR approach for correlative MCDM problems under 
Table 2 Summary of currently used similarity measures for IVIFSs

Author (s)

$\mathrm{Xu},(2007 \mathrm{a})$

$$
\begin{aligned}
& \operatorname{Sim}_{X_{1}}(R, S)=1-\frac{1}{4} \sum_{i=1}^{n} w_{i}\left(\begin{array}{c}
\left|\mu_{R}^{-}\left(u_{i}\right)-\mu_{S}^{-}\left(u_{i}\right)\right|+\left|v_{R}^{-}\left(u_{i}\right)-v_{S}^{-}\left(u_{i}\right)\right| \\
\left.+\left|\mu_{R}^{+}\left(u_{i}\right)-\mu_{S}^{+}\left(u_{i}\right)\right|+\left|v_{R}^{+}\left(u_{i}\right)-v_{S}^{+}\left(u_{i}\right)\right|\right)
\end{array}\right. \\
& \operatorname{Sim}_{X_{2}}(R, S)=1-\sqrt{\frac{1}{4} \sum_{i=1}^{n} w_{i}\left(\begin{array}{c}
\left(\mu_{R}^{-}\left(u_{i}\right)-\mu_{S}^{-}\left(u_{i}\right)\right)^{2}+\left(v_{R}^{-}\left(u_{i}\right)-v_{S}^{-}\left(u_{i}\right)\right)^{2} \\
+\left(\mu_{R}^{+}\left(u_{i}\right)-\mu_{S}^{+}\left(u_{i}\right)\right)^{2}+\left(v_{R}^{+}\left(u_{i}\right)-v_{S}^{+}\left(u_{i}\right)\right)^{2}
\end{array}\right)} \\
& \operatorname{Sim}_{X_{3}}(R, S)=1-\sum_{i=1}^{n} w_{i} \max \left(\begin{array}{l}
\left|\mu_{R}^{-}\left(u_{i}\right)-\mu_{S}^{-}\left(u_{i}\right)\right|,\left|v_{R}^{-}\left(u_{i}\right)-v_{S}^{-}\left(u_{i}\right)\right|, \\
\left|\mu_{R}^{+}\left(u_{i}\right)-\mu_{S}^{+}\left(u_{i}\right)\right|,\left|v_{R}^{+}\left(u_{i}\right)-v_{S}^{+}\left(u_{i}\right)\right|
\end{array}\right) \\
& \operatorname{Sim}_{X_{4}}(R, S)=1-\sqrt{\sum_{i=1}^{n} w_{i} \max \left(\begin{array}{c}
\left(\mu_{R}^{-}\left(u_{i}\right)-\mu_{S}^{-}\left(u_{i}\right)\right)^{2},\left(v_{R}^{-}\left(u_{i}\right)-v_{S}^{-}\left(u_{i}\right)\right)^{2}, \\
\left(\mu_{R}^{+}\left(u_{i}\right)-\mu_{S}^{+}\left(u_{i}\right)\right)^{2},\left(v_{R}^{+}\left(u_{i}\right)-v_{S}^{+}\left(u_{i}\right)\right)^{2}
\end{array}\right)}
\end{aligned}
$$

$\mathrm{Xu} \&$ Chen

(2008)

Wei et al.

(2011)

Ye (2012)
$\operatorname{Sim}_{X C_{1}}(R, S)=1-\left[\frac{1}{4 n} \sum_{i=1}^{n}\left(\begin{array}{c}\left|\mu_{R}^{-}\left(u_{i}\right)-\mu_{S}^{-}\left(u_{i}\right)\right|^{p}+\left|\mu_{R}^{+}\left(u_{i}\right)-\mu_{S}^{+}\left(u_{i}\right)\right|^{p} \\ +\left|v_{R}^{-}\left(u_{i}\right)-v_{S}^{-}\left(u_{i}\right)\right|^{p}+\left|v_{R}^{+}\left(u_{i}\right)-v_{S}^{+}\left(u_{i}\right)\right|^{p}\end{array}\right)\right]^{1 / p}, p>0$,

$\operatorname{Sim}_{X C_{2}}(R, S)=1-\left[\frac{1}{n} \sum_{i=1}^{n} \max \left(\begin{array}{c}\left|\mu_{R}^{-}\left(u_{i}\right)-\mu_{S}^{-}\left(u_{i}\right)\right|^{p},\left|\mu_{R}^{+}\left(u_{i}\right)-\mu_{S}^{+}\left(u_{i}\right)\right|^{p}, \\ \left|v_{R}^{-}\left(u_{i}\right)-v_{S}^{-}\left(u_{i}\right)\right|^{p},\left|v_{R}^{+}\left(u_{i}\right)-v_{S}^{+}\left(u_{i}\right)\right|^{p}\end{array}\right]^{1 / p}, p>0\right.$.

If let $p \rightarrow+\infty$ in $\operatorname{Sim}_{X C_{1}}(R, S)$ and $p=1$ in $\operatorname{Sim}_{X C_{2}}(R, S)$ then

$\operatorname{Sim}_{X C_{1}}^{\infty}(R, S)=1-\left[\max _{i}\left(\begin{array}{c}\left|\mu_{R}^{-}\left(u_{i}\right)-\mu_{S}^{-}\left(u_{i}\right)\right|,\left|\mu_{R}^{+}\left(u_{i}\right)-\mu_{S}^{+}\left(u_{i}\right)\right|, \\ \left|v_{R}^{-}\left(u_{i}\right)-v_{S}^{-}\left(u_{i}\right)\right|,\left|v_{R}^{+}\left(u_{i}\right)-v_{S}^{+}\left(u_{i}\right)\right|\end{array}\right)\right.$, ,

$\operatorname{Sim}_{X C_{2}}^{1}(R, S)=1-\left[\frac{1}{n} \sum_{i=1}^{n} \max \left(\begin{array}{c}\left|\mu_{R}^{-}\left(u_{i}\right)-\mu_{S}^{-}\left(u_{i}\right)\right|,\left|\mu_{R}^{+}\left(u_{i}\right)-\mu_{S}^{+}\left(u_{i}\right)\right|, \\ \left|v_{R}^{-}\left(u_{i}\right)-v_{S}^{-}\left(u_{i}\right)\right|,\left|v_{R}^{+}\left(u_{i}\right)-v_{S}^{+}\left(u_{i}\right)\right|\end{array}\right)\right]$,

$\operatorname{Sim}_{w}(R, S)=\frac{1}{n} \sum_{i=1}^{n} \frac{2-\min \left\{\mu_{i}^{-}, v_{i}^{-}\right\}-\min \left\{\mu_{i}^{+}, v_{i}^{+}\right\}}{2+\max \left\{\mu_{i}^{-}, v_{i}^{-}\right\}+\max \left\{\mu_{i}^{+}, v_{i}^{+}\right\}}$,

where $\mu_{i}^{-}=\left|\mu_{R}^{-}\left(u_{i}\right)-\mu_{S}^{-}\left(u_{i}\right)\right|, v_{i}^{-}=\left|v_{R}^{-}\left(u_{i}\right)-v_{S}^{-}\left(u_{i}\right)\right|$,

$\mu_{i}^{+}=\left|\mu_{R}^{+}\left(u_{i}\right)-\mu_{S}^{+}\left(u_{i}\right)\right|, v_{i}^{+}=\left|v_{R}^{+}\left(u_{i}\right)-v_{S}^{+}\left(u_{i}\right)\right|$.

$\operatorname{Sim}_{Y_{1}}(R, S)=\frac{1}{n} \sum_{i=1}^{n} \frac{2\left(\mu_{R} \mu_{S}+v_{R} v_{S}\right)}{\mu_{R}^{2}+v_{R}^{2}+\mu_{S}^{2}+v_{S}^{2}}$,

where $\mu_{R}=p_{1} \mu_{R}^{-}\left(u_{i}\right)+p_{2} \mu_{R}^{+}\left(u_{i}\right), v_{R}=q_{1} v_{R}^{-}\left(u_{i}\right)+q_{2} v_{R}^{+}\left(u_{i}\right)$,

$\mu_{S}=p_{1} \mu_{S}^{-}\left(u_{i}\right)+p_{2} \mu_{S}^{+}\left(u_{i}\right)$ and $v_{S}=q_{1} v_{S}^{-}\left(u_{i}\right)+q_{2} v_{S}^{+}\left(u_{i}\right)$, such that $p_{1}, p_{2}, q_{1}, q_{2} \in[0,1]$,

$p_{1}+p_{2}=1$ and $q_{1}+q_{2}=1$ are adjusting weight values 
Table 2 (continued)

\section{Author (s)}

Ye (2013)

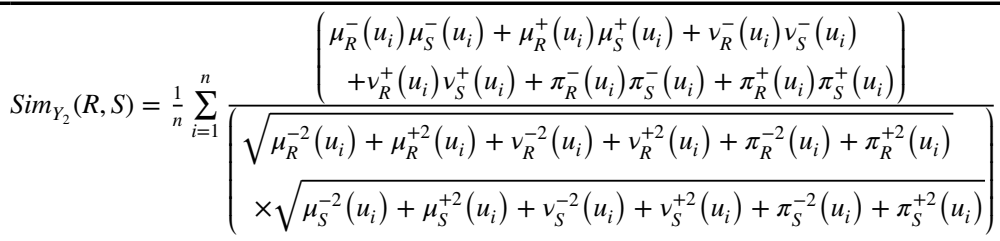

$$
\begin{aligned}
& \operatorname{Sim}_{H L_{1}}(R, S)=\frac{\sum_{i=1}^{n}\left\{\begin{array}{c}
2-\left(\left|\mu_{R}^{-}\left(u_{i}\right)-\mu_{S}^{-}\left(u_{i}\right)\right| \vee\left|v_{R}^{+}\left(u_{i}\right)-v_{S}^{+}\left(u_{i}\right)\right|\right)^{2} \\
-\left(\left|\mu_{R}^{-}\left(u_{i}\right)-\mu_{S}^{-}\left(u_{i}\right)\right| \vee\left|v_{R}^{+}\left(u_{i}\right)-v_{S}^{+}\left(u_{i}\right)\right|\right)
\end{array}\right\}}{\sum_{i=1}^{n}\left\{\begin{array}{c}
2+\left(\left|\mu_{R}^{-}\left(u_{i}\right)-\mu_{S}^{-}\left(u_{i}\right)\right| \vee\left|v_{R}^{+}\left(u_{i}\right)-v_{S}^{+}\left(u_{i}\right)\right|\right)^{3} \\
+\left(\left|\mu_{R}^{-}\left(u_{i}\right)-\mu_{S}^{-}\left(u_{i}\right)\right| \vee\left|v_{R}^{+}\left(u_{i}\right)-v_{S}^{+}\left(u_{i}\right)\right|\right)^{2}
\end{array}\right\}} \\
& \operatorname{Sim}_{H L_{2}}(R, S)=\frac{\sum_{i=1}^{n}\left\{\begin{array}{c}
16-2\left(\left|\mu_{R}^{-}\left(u_{i}\right)-\mu_{S}^{-}\left(u_{i}\right)\right| \vee\left|v_{R}^{+}\left(u_{i}\right)-v_{S}^{+}\left(u_{i}\right)\right|\right)^{2} \\
-2\left(\left|\mu_{R}^{-}\left(u_{i}\right)-\mu_{S}^{-}\left(u_{i}\right)\right| \vee\left|v_{R}^{+}\left(u_{i}\right)-v_{S}^{+}\left(u_{i}\right)\right|\right)
\end{array}\right\}}{\sum_{i=1}^{n}\left\{\begin{array}{c}
16+2\left(\left|\mu_{R}^{-}\left(u_{i}\right)-\mu_{S}^{-}\left(u_{i}\right)\right| \vee\left|v_{R}^{+}\left(u_{i}\right)-v_{S}^{+}\left(u_{i}\right)\right|\right)^{3} \\
+2\left(\left|\mu_{R}^{-}\left(u_{i}\right)-\mu_{S}^{-}\left(u_{i}\right)\right| \vee\left|v_{R}^{+}\left(u_{i}\right)-v_{S}^{+}\left(u_{i}\right)\right|\right)^{2}
\end{array}\right\}}
\end{aligned}
$$

Wu et al. (2014)

$$
\begin{aligned}
& \operatorname{Sim}_{w u}(R, S)=\frac{1}{n} \sum_{i=1}^{n} \frac{4-\left(\mu_{j}^{-}+\mu_{j}^{+}+v_{j}^{-}+v_{j}^{+}\right)+\left(\pi_{j}^{-}+\pi_{j}^{+}\right)}{4+\left(\mu_{j}^{-}+\mu_{j}^{+}+v_{j}^{-}+v_{j}^{+}\right)+\left(\pi_{j}^{-}+\pi_{j}^{+}\right)} \\
& \text {where } \mu_{j}^{\alpha}=\left|\mu_{R}^{\alpha}\left(u_{i}\right)-\mu_{S}^{\alpha}\left(u_{i}\right)\right|, v_{j}^{\alpha}=\left|v_{R}^{\alpha}\left(u_{i}\right)-v_{S}^{\alpha}\left(u_{i}\right)\right| \\
& \text { and } \pi_{j}^{\alpha}=\left|\pi_{R}^{\alpha}\left(u_{i}\right)-\pi_{S}^{\alpha}\left(u_{i}\right)\right| ; \alpha=\{-,+\}
\end{aligned}
$$

Meng and Chen (2016)

$\operatorname{Sim}_{M}(R, S)=\frac{1}{n} \sum_{i=1}^{n} \frac{4-\min \left\{\mu_{i}^{-}, v_{i}^{-}\right\}-\min \left\{\mu_{i}^{+}, v_{i}^{+}\right\}}{4+\max \left\{\mu_{i}^{-}, v_{i}^{-}\right\}+\max \left\{\mu_{i}^{+}, v_{i}^{+}\right\}}$,

where $\mu_{i}^{-}=\left|\mu_{R}^{-}\left(u_{i}\right)-\mu_{S}^{-}\left(u_{i}\right)\right|, v_{i}^{-}=\left|v_{R}^{-}\left(u_{i}\right)-v_{S}^{-}\left(u_{i}\right)\right|$,

$\mu_{i}^{+}=\left|\mu_{R}^{+}\left(u_{i}\right)-\mu_{S}^{+}\left(u_{i}\right)\right|, v_{i}^{+}=\left|v_{R}^{+}\left(u_{i}\right)-v_{S}^{+}\left(u_{i}\right)\right|$.

Pekala and
$\quad$ Balicki (2017) $\operatorname{Sim}_{P B_{1}}(R, S)=1-\frac{1}{n} \sum_{i=1}^{n} \max \left(\begin{array}{l}\left|\mu_{R}^{-}\left(u_{i}\right)-\mu_{S}^{-}\left(u_{i}\right)\right|,\left|v_{R}^{-}\left(u_{i}\right)-v_{S}^{-}\left(u_{i}\right)\right|, \\ \left|\mu_{R}^{+}\left(u_{i}\right)-\mu_{S}^{+}\left(u_{i}\right)\right|,\left|v_{R}^{+}\left(u_{i}\right)-v_{S}^{+}\left(u_{i}\right)\right|\end{array}\right)$

$\operatorname{Sim}_{P B_{2}}(R, S)=\operatorname{Inc}(R, S) \wedge \operatorname{Inc}(S, R)$, where

$\operatorname{Inc}(R, S)=\left\{\begin{array}{l}1, R=S=\emptyset \\ \frac{|R \wedge S|}{|R|}, \text { otherwise }\end{array}\right.$ and $|R|=\sum_{u_{i} \in U} \frac{\mu_{R}^{-}+\mu_{R}^{+}+2-v_{R}^{-}-v_{R}^{+}}{4}$

Rani et al.

(2018)

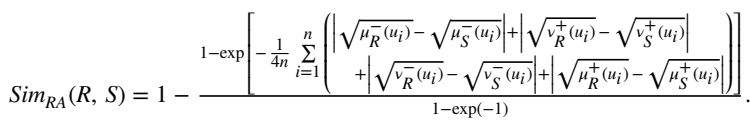


Author (s)

Mishra, and

Rani (2018a)

$$
\operatorname{Sim}_{M R_{1}}(R, S)=1-\left[\frac{1}{n(t+1) p} \sum_{i=1}^{n} \max \left(\begin{array}{l}
\mid\left(t \mu_{R}^{-}\left(u_{i}\right)-v_{R}^{-}\left(u_{i}\right)\right) \\
-\left(t \mu_{S}^{-}\left(u_{i}\right)-v_{S}^{-}\left(u_{i}\right)\right)
\end{array}|,| \begin{array}{c}
\left(t \mu_{R}^{+}\left(u_{i}\right)-v_{R}^{+}\left(u_{i}\right)\right) \\
-\left(t \mu_{S}^{+}\left(u_{i}\right)-v_{S}^{+}\left(u_{i}\right)\right)
\end{array}|,| \begin{array}{c}
\left(t v_{R}^{-}\left(u_{i}\right)-\mu_{R}^{-}\left(u_{i}\right)\right) \\
-\left(t v_{S}^{-}\left(u_{i}\right)-\mu_{S}^{-}\left(u_{i}\right)\right)
\end{array}|,| \begin{array}{c}
\left(t v_{R}^{+}\left(u_{i}\right)-\mu_{R}^{+}\left(u_{i}\right)\right) \\
-\left(t v_{S}^{+}\left(u_{i}\right)-\mu_{S}^{+}\left(u_{i}\right)\right)
\end{array} \mid\right)\right.
$$

where $t=1,2,3, \ldots$ categories the level of uncertainty and $p=1,2,3, \ldots$ be the $L_{p}-$ norm

Mishra and

Rani (2018b)

$\operatorname{Sim}_{M R_{2}}(R, S)=1-\frac{1-\exp \left[-\frac{1}{4 n} \sum_{i=1}^{n}\left(\begin{array}{c}\left|\mu_{R}^{-}\left(x_{i}\right)-\mu_{S}^{-}\left(x_{i}\right)\right|+\left|\mu_{R}^{+}\left(x_{i}\right)-\mu_{S}^{+}\left(x_{i}\right)\right| \\ +\left|v_{R}^{-}\left(x_{i}\right)-v_{S}^{-}\left(x_{i}\right)\right|+\left|v_{R}^{+}\left(x_{i}\right)-v_{S}^{+}\left(x_{i}\right)\right|\end{array}\right)\right]}{1-\exp (-1)}$.

IVIF environment. Mishra and Rani (2018a) proposed biparametric SM for IVIFSs to assess the plant location selection problem. Table 2 summarizes the earlier research works carried out into similarity measures for IVIFSs.

\subsubsection{New IVIF-similarity measures}

Based on Intarapaiboon (2014) for IFSs, we propose the following SMs for IVIFSs.

$$
\operatorname{Sim}_{1}(R, S)=\frac{\sum_{i=1}^{n}\left[\begin{array}{l}
\min \left\{\mu_{R}^{-}\left(u_{i}\right), \mu_{S}^{-}\left(u_{i}\right)\right\}+\min \left\{\mu_{R}^{+}\left(u_{i}\right), \mu_{S}^{+}\left(u_{i}\right)\right\} \\
+\min \left\{\left(1-v_{R}^{-}\left(u_{i}\right)\right),\left(1-v_{S}^{-}\left(u_{i}\right)\right)\right\}+\min \left\{\left(1-v_{R}^{+}\left(u_{i}\right)\right),\left(1-v_{S}^{+}\left(u_{i}\right)\right)\right\}
\end{array}\right]}{\sum_{i=1}^{n}\left[\begin{array}{l}
\max \left\{\mu_{R}^{-}\left(u_{i}\right), \mu_{S}^{-}\left(u_{i}\right)\right\}+\max \left\{\mu_{R}^{+}\left(u_{i}\right), \mu_{S}^{+}\left(u_{i}\right)\right\} \\
+\max \left\{\left(1-v_{R}^{-}\left(u_{i}\right)\right),\left(1-v_{S}^{-}\left(u_{i}\right)\right)\right\}+\max \left\{\left(1-v_{R}^{+}\left(u_{i}\right)\right),\left(1-v_{S}^{+}\left(u_{i}\right)\right)\right\}
\end{array}\right]} .
$$

Theorem 1: Function $\operatorname{Sim}_{1}(R, S)$ is valid $\operatorname{SM}$ for $\operatorname{IVIFSs}(U)$.

Proof $\left(S_{1}\right) \&\left(S_{2}\right)$ : Both are obvious from the definition of Eq. (8).

$\left(S_{3}\right): \quad$ Let $\quad \operatorname{Sim}_{1}(R, S) \cdot R, S \in \operatorname{IVIFSS}(U) \quad$ and $\quad R=S, \quad$ i.e., $\quad \mu_{R}^{-}\left(u_{i}\right)=\mu_{S}^{-}\left(u_{i}\right)$, $\mu_{R}^{+}\left(u_{i}\right)=\mu_{S}^{+}\left(u_{i}\right), v_{R}^{-}\left(u_{i}\right)=v_{S}^{-}\left(u_{i}\right)$ and $v_{R}^{+}\left(u_{i}\right)=v_{S}^{+}\left(u_{i}\right)$. Therefore, from Eq. (1), we obtain $\operatorname{Sim}_{1}(R, S)=1$.

Again, let $\operatorname{Sim}_{1}(R, S)=1$. Then, from Eq. (8), we have

$$
\begin{aligned}
& \sum_{i=1}^{n}\left[\begin{array}{l}
\min \left\{\mu_{R}^{-}\left(u_{i}\right), \mu_{S}^{-}\left(u_{i}\right)\right\}+\min \left\{\mu_{R}^{+}\left(u_{i}\right), \mu_{S}^{+}\left(u_{i}\right)\right\} \\
+\min \left\{\left(1-v_{R}^{-}\left(u_{i}\right)\right),\left(1-v_{S}^{-}\left(u_{i}\right)\right)\right\}+\min \left\{\left(1-v_{R}^{+}\left(u_{i}\right)\right),\left(1-v_{S}^{+}\left(u_{i}\right)\right)\right\}
\end{array}\right] \\
& =\sum_{i=1}^{n}\left[\begin{array}{l}
\max \left\{\mu_{R}^{-}\left(u_{i}\right), \mu_{S}^{-}\left(u_{i}\right)\right\}+\max \left\{\mu_{R}^{+}\left(u_{i}\right), \mu_{S}^{+}\left(u_{i}\right)\right\}+ \\
\max \left\{\left(1-v_{R}^{-}\left(u_{i}\right)\right),\left(1-v_{S}^{-}\left(u_{i}\right)\right)\right\}+\max \left\{\left(1-v_{R}^{+}\left(u_{i}\right)\right),\left(1-v_{S}^{+}\left(u_{i}\right)\right)\right\}
\end{array}\right]
\end{aligned}
$$

Since

$$
\forall_{u_{i} \in U}, \min \left\{\mu_{R}^{-}\left(u_{i}\right), \mu_{S}^{-}\left(u_{i}\right)\right\} \leq \max \left\{\mu_{R}^{-}\left(u_{i}\right), \mu_{S}^{-}\left(u_{i}\right)\right\}
$$




$$
\begin{aligned}
& \min \left\{\mu_{R}^{+}\left(u_{i}\right), \mu_{S}^{+}\left(u_{i}\right)\right\} \leq \max \left\{\mu_{R}^{+}\left(u_{i}\right), \mu_{S}^{+}\left(u_{i}\right)\right\}, \min \left\{\left(1-v_{R}^{-}\left(u_{i}\right)\right),\left(1-v_{S}^{-}\left(u_{i}\right)\right)\right\} \\
& \quad \leq \max \left\{\left(1-v_{R}^{-}\left(u_{i}\right)\right),\left(1-v_{S}^{-}\left(u_{i}\right)\right)\right\}
\end{aligned}
$$

and $\min \left\{\left(1-v_{R}^{+}\left(u_{i}\right)\right),\left(1-v_{S}^{+}\left(u_{i}\right)\right)\right\} \leq \max \left\{\left(1-v_{R}^{+}\left(u_{i}\right)\right),\left(1-v_{S}^{+}\left(u_{i}\right)\right)\right\} . \quad$ Therefore, Eq. (9) will be true when $\mu_{R}^{-}\left(u_{i}\right)=\mu_{S}^{-}\left(u_{i}\right), \mu_{R}^{+}\left(u_{i}\right)=\mu_{S}^{+}\left(u_{i}\right),\left(1-v_{R}^{-}\left(u_{i}\right)\right)=\left(1-v_{S}^{-}\left(u_{i}\right)\right)$ and $\left(1-v_{R}^{+}\left(u_{i}\right)\right)=\left(1-v_{S}^{+}\left(u_{i}\right)\right)$. It implies that $R=S$.

$\left(S_{4}\right)$ : Let $R, S, C \in I \operatorname{VIFSs}(U)$ and $R \subseteq S \subseteq C$, i.e., $\mu_{R}^{-}\left(u_{i}\right) \leq \mu_{S}^{-}\left(u_{i}\right) \leq \mu_{C}^{-}\left(u_{i}\right)$, $\mu_{R}^{+}\left(u_{i}\right) \leq \mu_{S}^{+}\left(u_{i}\right) \leq \mu_{C}^{+}\left(u_{i}\right), v_{R}^{-}\left(u_{i}\right) \geq v_{S}^{-}\left(u_{i}\right) \geq v_{C}^{-}\left(u_{i}\right)$ and $v_{R}^{+}\left(u_{i}\right) \geq v_{S}^{+}\left(u_{i}\right) \geq v_{C}^{+}\left(u_{i}\right)$, $\forall_{u_{i} \in U}$.

Again, $\forall_{u_{i} \in U},\left(1-v_{R}^{-}\left(u_{i}\right)\right) \leq\left(1-v_{S}^{-}\left(u_{i}\right)\right) \leq\left(1-v_{C}^{-}\left(u_{i}\right)\right)$

and $\left(1-v_{R}^{+}\left(u_{i}\right)\right) \leq\left(1-v_{S}^{+}\left(u_{i}\right)\right) \leq\left(1-v_{C}^{+}\left(u_{i}\right)\right)$.

Hence,

$$
\begin{gathered}
\operatorname{Sim}_{1}(R, C)=\frac{\sum_{i=1}^{n}\left[\begin{array}{l}
\min \left\{\mu_{R}^{-}\left(u_{i}\right), \mu_{C}^{-}\left(u_{i}\right)\right\}+\min \left\{\mu_{R}^{+}\left(u_{i}\right), \mu_{C}^{+}\left(u_{i}\right)\right\} \\
+\min \left\{\left(1-v_{R}^{-}\left(u_{i}\right)\right),\left(1-v_{C}^{-}\left(u_{i}\right)\right)\right\}+\min \left\{\left(1-v_{R}^{+}\left(u_{i}\right)\right),\left(1-v_{C}^{+}\left(u_{i}\right)\right)\right\}
\end{array}\right]}{\sum_{i=1}^{n}\left[\begin{array}{l}
\max \left\{\mu_{R}^{-}\left(u_{i}\right), \mu_{C}^{-}\left(u_{i}\right)\right\}+\max \left\{\mu_{R}^{+}\left(u_{i}\right), \mu_{C}^{+}\left(u_{i}\right)\right\} \\
+\max \left\{\left(1-v_{R}^{-}\left(u_{i}\right)\right),\left(1-v_{C}^{-}\left(u_{i}\right)\right)\right\}+\max \left\{\left(1-v_{R}^{+}\left(u_{i}\right)\right),\left(1-v_{C}^{+}\left(u_{i}\right)\right)\right\}
\end{array}\right]} \\
\operatorname{Sim}_{1}(R, C)=\frac{\sum_{i=1}^{n}\left[\mu_{R}^{-}\left(u_{i}\right)+\mu_{R}^{+}\left(u_{i}\right)+\left(1-v_{R}^{-}\left(u_{i}\right)\right)+\left(1-v_{R}^{+}\left(u_{i}\right)\right)\right]}{\sum_{i=1}^{n}\left[\mu_{C}^{-}\left(u_{i}\right)+\mu_{C}^{+}\left(u_{i}\right)+\left(1-v_{C}^{-}\left(u_{i}\right)\right)+\left(1-v_{C}^{+}\left(u_{i}\right)\right)\right]}
\end{gathered}
$$

Similarly

$$
\operatorname{Sim}_{1}(R, S)=\frac{\sum_{i=1}^{n}\left[\mu_{R}^{-}\left(u_{i}\right)+\mu_{R}^{+}\left(u_{i}\right)+\left(1-v_{R}^{-}\left(u_{i}\right)\right)+\left(1-v_{R}^{+}\left(u_{i}\right)\right)\right]}{\sum_{i=1}^{n}\left[\mu_{S}^{-}\left(u_{i}\right)+\mu_{S}^{+}\left(u_{i}\right)+\left(1-v_{S}^{-}\left(u_{i}\right)\right)+\left(1-v_{S}^{+}\left(u_{i}\right)\right)\right]}
$$

From Eqs. (10) and (11), we obtain

$$
\operatorname{Sim}_{1}(R, S) \geq \operatorname{Sim}_{1}(R, C) .
$$

On a similar line, we obtain $\operatorname{Sim}_{1}(S, C) \geq \operatorname{Sim}_{1}(R, C)$.

After that, another IVIF-SM for IVIFSs, which is introduced from combining $\operatorname{Sim}_{1}(R, S)$, and a notion of SMs and lattice, is presented. Generally, a lattice of a nonempty function is based on a hierarchical structure prepared by a partial order such that, for every two objects in the lattice, a lub (supremum) and a glb (infimum) exist. Within a lattice, similarity for two conceptions within is generally evaluated through the use of information of their supremum and infimum, for instance, the depth from the root to the supremum.

Through utilizing the IVIFSs as the subset relationship and a lattice concept that is given in Eq. (8) as a partial order, it is possible to assemble a lattice. For any pair of IVIFSs, the infimum and supremum can be computed from intersection and union, respectively. Thus, the similarity measure is given by 


$$
\operatorname{Sim}_{2}(R, S)=\sqrt{\operatorname{Sim}_{1}\left(R, C_{R S}\right) \times \operatorname{Sim}_{1}\left(S, C_{R S}\right)},
$$

where $C_{R S}=R \cup S$ and $\operatorname{Sim}_{1}(R, S)$ is given by Eq. (8).

Theorem 2 Mapping $\operatorname{Sim}_{2}(R, S)$ is a valid SM for IVIFSs.

Proof $\left(S_{1}\right) \&\left(S_{2}\right)$ : Both are straightforward.

$\left(S_{3}\right)$ : Let $R, S \in I V I F S s$ and $R=S$, Since $C_{R S}=R \cup S$. Therefore $R=S=C_{R S}$ and $\operatorname{Sim}_{1}(R, S)$ is satisfied $\left(S_{3}\right)$. Hence $\operatorname{Sim}_{1}(R, S)=1$. Again let $\operatorname{Sim}_{2}(R, S)=1$. Therefore, $\operatorname{Sim}_{1}\left(R, C_{R S}\right)=\operatorname{Sim}_{1}\left(S, C_{R S}\right)=1$, when $C_{R S}=R \cup S$ and $\operatorname{Sim}_{1}(R, S)$ satisfies $\left(S_{3}\right)$. Hence, $R=S=C_{R S}$.

$\left(S_{4}\right)$ : Let $R, S, C \in \operatorname{IVIFSS}(U)$ and $R \subseteq S \subseteq C$. Then $R \cup S=S, R \cup C=C$ and $S \cup C=C$.

$$
\begin{gathered}
\text { Now } \operatorname{Sim}_{2}(R, C)=\sqrt{\operatorname{Sim}_{1}\left(R, C_{R C}\right) \times \operatorname{Sim}_{1}\left(C, C_{R C}\right)} \\
\qquad \operatorname{Sim}_{2}(R, C)=\sqrt{\operatorname{Sim}_{1}(R, C) \times \operatorname{Sim}_{1}(C, C)} \\
\operatorname{Sim}_{2}(R, C)=\sqrt{\operatorname{Sim}_{1}(R, C)}
\end{gathered}
$$

On a similar line, $\operatorname{Sim}_{2}(R, S)=\sqrt{\operatorname{Sim}_{1}(R, S)}$.

Since $\operatorname{Sim}_{1}(R, S)$ holds $\left(S_{4}\right)$, i.e., $\operatorname{Sim}_{1}(R, S) \geq \operatorname{Sim}_{1}(R, C), \operatorname{Sim}_{2}(R, S) \geq \operatorname{Sim}_{2}(R, C)$.

Similarity, $\operatorname{Sim}_{2}(S, C) \geq \operatorname{Sim}_{2}(R, C)$. [Proved].

Here, if we take $R$ and $S$ as intervals illustration, the information conceded by them is obtained by not only in the terms of bounds (lower and upper), but also the length of the interval. Based on Song et al. (2014), we extend the following similarity measure for IVIFSs as

$$
\begin{aligned}
& \operatorname{Sim}_{3}(R, S)=\frac{1}{4 n} \sum_{i=1}^{n}\left[\sqrt{\mu_{R}^{-}\left(u_{i}\right) \mu_{S}^{-}\left(u_{i}\right)}+\sqrt{\mu_{R}^{+}\left(u_{i}\right) \mu_{S}^{+}\left(u_{i}\right)}+2 \sqrt{v_{R}^{-}\left(u_{i}\right) v_{S}^{-}\left(u_{i}\right)}\right. \\
& +2 \sqrt{v_{R}^{+}\left(u_{i}\right) v_{S}^{+}\left(u_{i}\right)}+\sqrt{\pi_{R}^{-}\left(u_{i}\right) \pi_{S}^{-}\left(u_{i}\right)}+\sqrt{\pi_{R}^{+}\left(u_{i}\right) \pi_{S}^{+}\left(u_{i}\right)} \\
& \left.+\sqrt{\left(1-v_{R}^{-}\left(u_{i}\right)\right)\left(1-v_{S}^{-}\left(u_{i}\right)\right)}+\sqrt{\left(1-v_{R}^{+}\left(u_{i}\right)\right)\left(1-v_{S}^{+}\left(u_{i}\right)\right)}\right]
\end{aligned}
$$

Theorem 3 Function $\operatorname{Sim}_{3}(R, S)$ given by Eq. (13) is valid SM for IVIFSs $(U)$.

Proof (S1): For each $x, y \in[0, \infty]$, we obtain.

$$
0 \leq \sqrt{x y} \leq \frac{x+y}{2} .
$$

Since $\quad 0 \leq \mu_{R}^{-}\left(u_{i}\right), \mu_{R}^{+}\left(u_{i}\right) \leq 1,0 \leq v_{R}^{-}\left(u_{i}\right), \nu_{R}^{+}\left(u_{i}\right) \leq 10 \leq \pi_{R}^{-}\left(u_{i}\right), \pi_{R}^{+}\left(u_{i}\right) \leq 1$, $0 \leq \mu_{S}^{-}\left(u_{i}\right), \quad \mu_{S}^{+}\left(u_{i}\right) \leq 1, \quad 0 \leq v_{S}^{-}\left(u_{i}\right), \quad v_{S}^{+}\left(u_{i}\right) \leq 1, \quad 0 \leq \pi_{S}^{-}\left(u_{i}\right), \quad \pi_{S}^{+}\left(u_{i}\right) \leq 1$, $0 \leq\left(1-v_{R}^{-}\left(u_{i}\right)\right),\left(1-v_{R}^{+}\left(u_{i}\right)\right) \leq 1$ and $0 \leq\left(1-v_{S}^{-}\left(u_{i}\right)\right),\left(1-v_{S}^{+}\left(u_{i}\right)\right) \leq 1$, we have 


$$
\begin{gathered}
0 \leq \sqrt{\mu_{R}^{-}\left(u_{i}\right) \mu_{S}^{-}\left(u_{i}\right)} \leq \frac{\mu_{R}^{-}\left(u_{i}\right)+\mu_{S}^{-}\left(u_{i}\right)}{2}, 0 \leq \sqrt{v_{R}^{-}\left(u_{i}\right) v_{S}^{-}\left(u_{i}\right)} \leq \frac{v_{R}^{-}\left(u_{i}\right)+v_{S}^{-}\left(u_{i}\right)}{2}, \\
0 \leq \sqrt{\mu_{R}^{+}\left(u_{i}\right) \mu_{S}^{+}\left(u_{i}\right)} \leq \frac{\mu_{R}^{+}\left(u_{i}\right)+\mu_{S}^{+}\left(u_{i}\right)}{2}, 0 \leq \sqrt{v_{R}^{+}\left(u_{i}\right) v_{S}^{+}\left(u_{i}\right)} \leq \frac{v_{R}^{+}\left(u_{i}\right)+v_{S}^{+}\left(u_{i}\right)}{2}, \\
0 \leq \sqrt{\pi_{R}^{-}\left(u_{i}\right) \pi_{S}^{-}\left(u_{i}\right)} \leq \frac{\pi_{R}^{-}\left(u_{i}\right)+\pi_{S}^{-}\left(u_{i}\right)}{2}, 0 \leq \sqrt{\pi_{R}^{+}\left(u_{i}\right) \pi_{S}^{+}\left(u_{i}\right)} \leq \frac{\pi_{R}^{+}\left(u_{i}\right)+\pi_{S}^{+}\left(u_{i}\right)}{2}, \\
0 \leq \sqrt{\left(1-v_{R}^{-}\left(u_{i}\right)\right)\left(1-v_{S}^{-}\left(u_{i}\right)\right)} \leq \frac{\left(1-v_{R}^{-}\left(u_{i}\right)\right)+\left(1-v_{S}^{-}\left(u_{i}\right)\right)}{2} \\
0 \leq \sqrt{\left(1-v_{R}^{+}\left(u_{i}\right)\right)\left(1-v_{S}^{+}\left(u_{i}\right)\right)} \leq \frac{\left(1-v_{R}^{+}\left(u_{i}\right)\right)+\left(1-v_{S}^{+}\left(u_{i}\right)\right)}{2}
\end{gathered}
$$

Now,

$$
\begin{gathered}
0 \leq\left[\sqrt{\mu_{R}^{-}\left(u_{i}\right) \mu_{S}^{-}\left(u_{i}\right)}+\sqrt{\mu_{R}^{+}\left(u_{i}\right) \mu_{S}^{+}\left(u_{i}\right)}+2 \sqrt{v_{R}^{-}\left(u_{i}\right) v_{S}^{-}\left(u_{i}\right)}\right. \\
+2 \sqrt{v_{R}^{+}\left(u_{i}\right) v_{S}^{+}\left(u_{i}\right)}+\sqrt{\pi_{R}^{-}\left(u_{i}\right) \pi_{S}^{-}\left(u_{i}\right)}+\sqrt{\pi_{R}^{+}\left(u_{i}\right) \pi_{S}^{+}\left(u_{i}\right)} \\
\left.+\sqrt{\left(1-v_{R}^{-}\left(u_{i}\right)\right)\left(1-v_{S}^{-}\left(u_{i}\right)\right)}+\sqrt{\left(1-v_{R}^{+}\left(u_{i}\right)\right)\left(1-v_{S}^{+}\left(u_{i}\right)\right)}\right] \\
\leq \frac{\mu_{R}^{-}\left(u_{i}\right)+\mu_{S}^{-}\left(u_{i}\right)}{2}+\frac{\mu_{R}^{+}\left(u_{i}\right)+\mu_{S}^{+}\left(u_{i}\right)}{2}+2 \frac{v_{R}^{-}\left(u_{i}\right)+v_{S}^{-}\left(u_{i}\right)}{2}+2 \frac{v_{R}^{+}\left(u_{i}\right)+v_{S}^{+}\left(u_{i}\right)}{2} \\
+\frac{\pi_{R}^{-}\left(u_{i}\right)+\pi_{S}^{-}\left(u_{i}\right)}{2}+\frac{\pi_{R}^{+}\left(u_{i}\right)+\pi_{S}^{+}\left(u_{i}\right)}{2}+\frac{\left(1-v_{R}^{-}\left(u_{i}\right)\right)+\left(1-v_{S}^{-}\left(u_{i}\right)\right)}{2} \\
+\frac{\mu_{S}^{+}\left(u_{i}\right)+v_{S}^{+}\left(u_{i}\right)+\pi_{S}^{+}\left(u_{i}\right)}{2}=4, v_{u_{i} \in U} \cdot \\
=2+\frac{\mu_{R}^{-}\left(u_{i}\right)+v_{R}^{-}\left(u_{i}\right)+\pi_{R}^{-}\left(u_{i}\right)}{2}+\frac{\mu_{R}^{+}\left(u_{i}\right)+v_{R}^{+}\left(u_{i}\right)+\pi_{R}^{+}\left(u_{i}\right)}{2}+\frac{\mu_{S}^{-}\left(u_{i}\right)+v_{S}^{-}\left(u_{i}\right)+\pi_{S}^{-}\left(u_{i}\right)}{2}
\end{gathered}
$$

Hence, 


$$
\begin{aligned}
& 0 \leq \frac{1}{4 n} \sum_{i=1}^{n}\left[\sqrt{\mu_{R}^{-}\left(u_{i}\right) \mu_{S}^{-}\left(x_{i}\right)}+\sqrt{\mu_{R}^{+}\left(u_{i}\right) \mu_{S}^{+}\left(u_{i}\right)}+2 \sqrt{v_{R}^{-}\left(u_{i}\right) v_{S}^{-}\left(u_{i}\right)}\right. \\
& +2 \sqrt{v_{R}^{+}\left(u_{i}\right) v_{S}^{+}\left(u_{i}\right)}+\sqrt{\pi_{R}^{-}\left(u_{i}\right) \pi_{S}^{-}\left(u_{i}\right)}+\sqrt{\pi_{R}^{+}\left(u_{i}\right) \pi_{S}^{+}\left(u_{i}\right)} \\
& \left.+\sqrt{\left(1-v_{R}^{-}\left(u_{i}\right)\right)\left(1-v_{S}^{-}\left(u_{i}\right)\right)}+\sqrt{\left(1-v_{R}^{+}\left(u_{i}\right)\right)\left(1-v_{S}^{+}\left(u_{i}\right)\right)}\right] \leq 1 .
\end{aligned}
$$

Thus, $0 \leq \operatorname{Sim}_{3}(R, S) \leq 1$.

$\left(S_{2}\right)$ : It is obvious from the definition.

$\left(S_{3}\right)$ : From Eq. (14), $\sqrt{x y}$ has its maximum degree $\frac{(x+y)}{2}$, when $x=y$. As a result, let $\operatorname{Sim}_{3}(R, S)=1$

$$
\begin{gathered}
\Leftrightarrow\left[\sqrt{\mu_{R}^{-}\left(u_{i}\right) \mu_{S}^{-}\left(u_{i}\right)}+\sqrt{\mu_{R}^{+}\left(u_{i}\right) \mu_{S}^{+}\left(u_{i}\right)}+2 \sqrt{v_{R}^{-}\left(u_{i}\right) v_{S}^{-}\left(u_{i}\right)}\right. \\
+2 \sqrt{v_{R}^{+}\left(u_{i}\right) v_{S}^{+}\left(u_{i}\right)}+\sqrt{\pi_{R}^{-}\left(u_{i}\right) \pi_{S}^{-}\left(u_{i}\right)}+\sqrt{\pi_{R}^{+}\left(u_{i}\right) \pi_{S}^{+}\left(u_{i}\right)} \\
\left.+\sqrt{\left(1-v_{R}^{-}\left(u_{i}\right)\right)\left(1-v_{S}^{-}\left(u_{i}\right)\right)}+\sqrt{\left(1-v_{R}^{+}\left(u_{i}\right)\right)\left(1-v_{S}^{+}\left(u_{i}\right)\right)}\right]=4 ; \forall_{u_{i} \in U} . \\
\Leftrightarrow \mu_{R}^{-}\left(u_{i}\right)=\mu_{S}^{-}\left(u_{i}\right), \mu_{R}^{+}\left(u_{i}\right)=\mu_{S}^{+}\left(u_{i}\right), v_{R}^{-}\left(u_{i}\right)=v_{S}^{-}\left(u_{i}\right), v_{R}^{+}\left(u_{i}\right)=v_{S}^{+}\left(u_{i}\right), \\
\pi_{R}^{-}\left(u_{i}\right)=\pi_{S}^{-}\left(u_{i}\right), \pi_{R}^{+}\left(u_{i}\right)=\pi_{S}^{+}\left(u_{i}\right),\left(1-v_{R}^{-}\left(u_{i}\right)\right)=\left(1-v_{S}^{-}\left(u_{i}\right)\right) \text { and } \\
\left(1-v_{R}^{+}\left(u_{i}\right)\right)=\left(1-v_{S}^{+}\left(u_{i}\right)\right) . \\
\Leftrightarrow R=S .
\end{gathered}
$$

Hence,

$\operatorname{Sim}_{3}(R, S)=1$ iff $R=S$.

$\left(S_{4}\right)$ : Let $R, S, C \in \operatorname{IVIFSs}(U)$ and $R \subseteq S \subseteq C$, i.e., $0 \leq \mu_{R}^{-}\left(u_{i}\right) \leq \mu_{S}^{-}\left(u_{i}\right) \leq \mu_{C}^{-}\left(u_{i}\right) \leq 1$, $0 \leq \mu_{R}^{+}\left(u_{i}\right) \leq \mu_{S}^{+}\left(u_{i}\right) \leq \mu_{C}^{+}\left(u_{i}\right) \leq 1,0 \leq v_{R}^{-}\left(u_{i}\right) \leq v_{S}^{-}\left(u_{i}\right) \leq v_{C}^{-}\left(u_{i}\right) \leq 1,0 \leq v_{R}^{+}\left(u_{i}\right) \leq v_{S}^{+}$ $\left(u_{i}\right) \leq v_{C}^{+}\left(u_{i}\right) \leq 1$.

Therefore, from Eq. (13), we obtain

$$
\begin{aligned}
& \operatorname{Sim}_{3}(A, C)=\frac{1}{4 n} \sum_{i=1}^{n}\left[\sqrt{\mu_{R}^{-}\left(u_{i}\right) \mu_{C}^{-}\left(u_{i}\right)}+\sqrt{\mu_{R}^{+}\left(u_{i}\right) \mu_{C}^{+}\left(u_{i}\right)}+2 \sqrt{v_{R}^{-}\left(u_{i}\right) v_{C}^{-}\left(u_{i}\right)}\right. \\
& +2 \sqrt{v_{R}^{+}\left(u_{i}\right) v_{C}^{+}\left(u_{i}\right)}+\sqrt{\pi_{R}^{-}\left(u_{i}\right) \pi_{C}^{-}\left(u_{i}\right)}+\sqrt{\pi_{R}^{+}\left(u_{i}\right) \pi_{C}^{+}\left(u_{i}\right)} \\
& \left.+\sqrt{\left(1-v_{R}^{-}\left(u_{i}\right)\right)\left(1-v_{C}^{-}\left(u_{i}\right)\right)}+\sqrt{\left(1-v_{R}^{+}\left(u_{i}\right)\right)\left(1-v_{C}^{+}\left(u_{i}\right)\right)}\right]
\end{aligned}
$$




$$
\begin{aligned}
& \operatorname{Sim}_{3}(B, C)=\frac{1}{4 n} \sum_{i=1}^{n}\left[\sqrt{\mu_{S}^{-}\left(u_{i}\right) \mu_{C}^{-}\left(u_{i}\right)}+\sqrt{\mu_{S}^{+}\left(u_{i}\right) \mu_{C}^{+}\left(u_{i}\right)}+2 \sqrt{v_{S}^{-}\left(u_{i}\right) v_{C}^{-}\left(u_{i}\right)}\right. \\
& +2 \sqrt{v_{S}^{+}\left(u_{i}\right) v_{C}^{+}\left(u_{i}\right)}+\sqrt{\pi_{S}^{-}\left(u_{i}\right) \pi_{C}^{-}\left(u_{i}\right)}+\sqrt{\pi_{S}^{+}\left(u_{i}\right) \pi_{C}^{+}\left(u_{i}\right)} \\
& \left.+\sqrt{\left(1-v_{S}^{-}\left(u_{i}\right)\right)\left(1-v_{C}^{-}\left(u_{i}\right)\right)}+\sqrt{\left(1-v_{S}^{+}\left(u_{i}\right)\right)\left(1-v_{C}^{+}\left(u_{i}\right)\right)}\right]
\end{aligned}
$$

For $a_{1}, a_{2}, b_{1}, b_{2} \in[0,1], a_{1}+b_{1} \leq 1$ and $a_{2}+b_{2} \leq 1$, we define a function $F$ as

$$
F\left(x_{1}, x_{2}, y_{1}, y_{2}\right) \equiv f\left(x_{1}, y_{1}\right)+g\left(x_{2}, y_{2}\right),
$$

where

$$
f\left(x_{1}, y_{1}\right)=\left[\sqrt{a_{1} x_{1}}+2 \sqrt{b_{1} y_{1}}+\sqrt{\left(1-a_{1}-b_{1}\right)\left(1-x_{1}-y_{1}\right)}+\sqrt{\left(1-b_{1}\right)\left(1-y_{1}\right)}\right]
$$

and

$$
g\left(x_{2}, y_{2}\right)=\left[\sqrt{a_{2} x_{2}}+2 \sqrt{b_{2} y_{2}}+\sqrt{\left(1-a_{2}-b_{2}\right)\left(1-x_{2}-y_{2}\right)}+\sqrt{\left(1-b_{2}\right)\left(1-y_{2}\right)}\right]
$$

such that $x_{1}, x_{2}, y_{1}, y_{2} \in[0,1], x_{1}+y_{1}, x_{2}+y_{2} \in[0,1]$.

Now,

$$
\frac{\partial f}{\partial x_{1}}=\frac{\sqrt{a_{1}}}{2 \sqrt{x_{1}}}-\frac{\sqrt{\left(1-a_{1}-b_{1}\right)}}{2 \sqrt{1-x_{1}-y_{1}}} \text { and } \frac{\partial f}{\partial y_{1}}=\frac{\sqrt{b_{1}}}{\sqrt{y_{1}}}-\frac{\sqrt{\left(1-a_{1}-b_{1}\right)}}{2 \sqrt{1-x_{1}-y_{1}}}-\frac{\sqrt{1-b_{1}}}{2 \sqrt{1-y_{1}}}
$$

Given that $a_{1} \leq x_{1} \leq 1 b_{1} \leq 1$, then we get.

$$
\left.\frac{\partial f}{\partial x_{1}}\right|_{y_{1}=b_{1}}=\frac{\sqrt{a_{1}}}{2 \sqrt{x_{1}}}-\frac{\sqrt{\left(1-a_{1}-b_{1}\right)}}{2 \sqrt{1-x_{1}-y_{1}}}=\frac{\left(a_{1}-x_{1}\right)\left(1-b_{1}\right)}{2 \sqrt{x_{1}\left(1-x_{1}-y_{1}\right)}\left(\sqrt{a_{1}\left(1-x_{1}-y_{1}\right)}+\sqrt{x_{1}\left(1-a_{1}-b_{1}\right)}\right)}
$$

$\left.\frac{\partial f}{\partial x_{1}}\right|_{y_{1}=b_{1}} \leq 0$, that means $f$ is a decreasing mapping of $x_{1}$ when $y_{1}=b_{1}$. For $0 \leq x_{1} \leq a_{1}, b_{1} \leq 1$, we obtain $\left(\frac{\partial f}{\partial x_{1}}\right)_{y_{1}=b_{1}} \geq 0$, i.e., $f$ is an increasing mapping of $x_{1}$ ,when $x_{1} \leq a_{1}$ and $y_{1}=b_{1}$.

Again, for $\frac{\partial f}{\partial y_{1}}$, we get

$$
\left.\frac{\partial f}{\partial y_{1}}\right|_{x_{1}=a_{1}}=\frac{\left(b_{1}-y_{1}\right)\left(1-a_{1}\right)}{2 \sqrt{y_{1}\left(1-x_{1}-y_{1}\right)}\left(\sqrt{b_{1}\left(1-x_{1}-y_{1}\right)}+\sqrt{y_{1}\left(1-a_{1}-b_{1}\right)}\right)}+\frac{\left(b_{1}-y_{1}\right)}{2 \sqrt{y_{1}\left(1-y_{1}\right)}\left(\sqrt{b_{1}\left(1-y_{1}\right)}+\sqrt{y_{1}\left(1-b_{1}\right)}\right)}
$$

which provides that $f$ is an increasing mapping of $y_{1}$ for $x_{1}=a_{1}, y_{1} \leq b_{1}$, but decreasing mapping when $x_{1}=a_{1}, y_{1} \geq b_{1}$.

Similarly, 


$$
\frac{\partial g}{\partial x_{2}}=\frac{\sqrt{a_{2}}}{2 \sqrt{x_{2}}}-\frac{\sqrt{\left(1-a_{2}-b_{2}\right)}}{2 \sqrt{1-x_{2}-y_{2}}} \text { and } \frac{\partial g}{\partial y_{2}}=\frac{\sqrt{b_{2}}}{\sqrt{y_{2}}}-\frac{\sqrt{\left(1-a_{2}-b_{2}\right)}}{2 \sqrt{1-x_{2}-y_{2}}}-\frac{\sqrt{1-b_{2}}}{2 \sqrt{1-y_{2}}}
$$

From Eq. (23), In $0 \leq a_{2} \leq x_{2} \leq 1$ and $b_{2} \leq 1, g$ is a decreasing function of $x_{2}$ at $y_{2}=b_{2}$, in $x_{2} \leq a_{2}, g$ is an increasing function of $x_{2}$ at $y_{2}=b_{2}$. On a similar manner, $g$ is a decreasing function of $y_{2}$ for $x_{2}=a_{2}, y_{2} \geq b_{2}$, but an increasing function when $x_{2}=a_{2}, y_{2} \leq b_{2}$.

Given that $a_{1}=\mu_{R}^{-}\left(u_{i}\right), a_{2}=\mu_{R}^{+}\left(u_{i}\right), b_{1}=v_{R}^{-}\left(u_{i}\right), b_{2}=v_{R}^{+}\left(u_{i}\right)$, satisfying

$$
a_{1}=\mu_{R}^{-}\left(u_{i}\right) \leq \mu_{S}^{-}\left(u_{i}\right) \leq \mu_{C}^{-}\left(u_{i}\right)
$$

$a_{2}=\mu_{R}^{+}\left(u_{i}\right) \leq \mu_{S}^{+}\left(u_{i}\right) \leq \mu_{C}^{+}\left(u_{i}\right)$, $v_{C}^{+}\left(u_{i}\right) \leq v_{S}^{+}\left(u_{i}\right) \leq v_{R}^{+}\left(u_{i}\right)=b_{2}$, we obtain

$$
v_{C}^{-}\left(u_{i}\right) \leq v_{S}^{-}\left(u_{i}\right) \leq v_{R}^{-}\left(u_{i}\right)=b_{1},
$$

$$
\begin{aligned}
& f\left(\mu_{C}^{-}\left(x_{i}\right), b_{1}\right) \leq f\left(\mu_{B}^{-}\left(x_{i}\right), b_{1}\right) \leq f\left(a_{1}, b_{1}\right) \\
& g\left(\mu_{C}^{+}\left(x_{i}\right), b_{2}\right) \leq g\left(\mu_{B}^{+}\left(x_{i}\right), b_{2}\right) \leq g\left(a_{2}, b_{2}\right) \\
& f\left(a_{1}, v_{C}^{-}\left(x_{i}\right)\right) \leq f\left(a_{1}, v_{B}^{-}\left(x_{i}\right)\right) \leq f\left(a_{1}, b_{1}\right) \\
& g\left(a_{2}, v_{C}^{+}\left(x_{i}\right)\right) \leq g\left(a_{2}, v_{B}^{+}\left(x_{i}\right)\right) \leq g\left(a_{2}, b_{2}\right) .
\end{aligned}
$$

Since $f\left(x_{1}, y_{1}\right)$ and $g\left(x_{2}, y_{2}\right)$ continues concave function,

$$
f\left(\mu_{C}^{-}\left(u_{i}\right), \nu_{C}^{-}\left(u_{i}\right)\right) \leq f\left(\mu_{S}^{-}\left(u_{i}\right), \nu_{S}^{-}\left(u_{i}\right)\right), g\left(\mu_{C}^{+}\left(u_{i}\right), \nu_{C}^{+}\left(u_{i}\right)\right) \leq g\left(\mu_{S}^{+}\left(u_{i}\right), \nu_{S}^{+}\left(u_{i}\right)\right) .
$$

Therefore, $F=f\left(x_{1}, y_{1}\right)+g\left(x_{2}, y_{2}\right)$ is also a continuous concave mapping.

Now,

Table 3 Comparison of different IVIF-SMs under various counter-intuitive cases

\begin{tabular}{lllll}
\hline$R_{i} S_{i}$ & $([0.2,0.3],[0.4,0.6])$ & $([0.2,0.3],[0.4,0.6])$ & $([0.2,0.3],[0.3,0.5])$ & $([0.2,0.3],[0.3,0.5])$ \\
& $([0.3,0.4],[0.4,0.6])$ & $([0.3,0.4],[0.3,0.5])$ & $([0.3,0.4],[0.4,0.6])$ & $([0.3,0.4],[0.3,0.5])$ \\
\hline $\operatorname{Sim}_{X C_{1}}(R, S)$ & $\mathbf{0 . 9 0}$ & $\mathbf{0 . 9 0}$ & $\mathbf{0 . 9 0}$ & 0.95 \\
$\operatorname{Sim}_{X C_{2}}(R, S)$ & $\mathbf{0 . 9 0}$ & $\mathbf{0 . 9 0}$ & $\mathbf{0 . 9 0}$ & $\mathbf{0 . 9 0}$ \\
$\operatorname{Sim}_{W}(R, S)$ & $\mathbf{0 . 9 0 9 1}$ & $\mathbf{0 . 8 1 8 2}$ & $\mathbf{0 . 8 1 8 2}$ & $\mathbf{0 . 9 0 9 1}$ \\
$\operatorname{Sim}_{Y_{2}}(R, S)$ & 0.9765 & 0.9754 & 0.9277 & 0.9737 \\
$\operatorname{Sim}_{W u}(R, S)$ & $\mathbf{0 . 9 0 4 8}$ & $\mathbf{0 . 8 1 8 2}$ & $\mathbf{0 . 8 1 8 2}$ & $\mathbf{0 . 9 0 4 8}$ \\
$\operatorname{Sim}_{M}(R, S)$ & $\mathbf{0 . 9 5 2 4}$ & $\mathbf{0 . 9 0 4 8}$ & $\mathbf{0 . 9 0 4 8}$ & $\mathbf{0 . 9 5 2 4}$ \\
$\operatorname{Sim}_{P B_{1}}(R, S)$ & $\mathbf{0 . 9 0 0 0}$ & $\mathbf{0 . 9 0 0 0}$ & $\mathbf{0 . 9 0 0 0}$ & $\mathbf{0 . 9 0 0 0}$ \\
$\operatorname{Sim}_{M R_{2}}(R, S)$ & $\mathbf{0 . 9 2 2 8}$ & $\mathbf{0 . 8 4 9 5}$ & $\mathbf{0 . 8 4 9 5}$ & $\mathbf{0 . 9 2 2 8}$ \\
$\operatorname{Sim}_{1}(R, S)$ & 0.8824 & $\mathbf{0 . 7 8 9 5}$ & $\mathbf{0 . 7 8 9 5}$ & 0.8947 \\
$\operatorname{Sim}_{3}(R, S)$ & 0.9844 & 0.9937 & 0.9656 & 0.9950 \\
\hline
\end{tabular}

Bold character indicates counter-intuitive cases 


$$
\begin{gathered}
\quad\left[\sqrt{\mu_{R}^{-}\left(u_{i}\right) \mu_{C}^{-}\left(u_{i}\right)}+\sqrt{\mu_{R}^{+}\left(u_{i}\right) \mu_{C}^{+}\left(u_{i}\right)}+2 \sqrt{v_{R}^{-}\left(u_{i}\right) v_{C}^{-}\left(u_{i}\right)}\right. \\
+2 \sqrt{v_{R}^{+}\left(u_{i}\right) v_{C}^{+}\left(u_{i}\right)}+\sqrt{\pi_{R}^{-}\left(u_{i}\right) \pi_{C}^{-}\left(u_{i}\right)}+\sqrt{\pi_{R}^{+}\left(u_{i}\right) \pi_{C}^{+}\left(u_{i}\right)} \\
\left.+\sqrt{\left(1-v_{R}^{-}\left(u_{i}\right)\right)\left(1-v_{C}^{-}\left(u_{i}\right)\right)}+\sqrt{\left(1-v_{R}^{+}\left(u_{i}\right)\right)\left(1-v_{C}^{+}\left(u_{i}\right)\right)}\right] \\
\leq\left[\sqrt{\mu_{S}^{-}\left(u_{i}\right) \mu_{C}^{-}\left(u_{i}\right)}+\sqrt{\mu_{S}^{+}\left(u_{i}\right) \mu_{C}^{+}\left(u_{i}\right)}+2 \sqrt{v_{S}^{-}\left(u_{i}\right) v_{C}^{-}\left(u_{i}\right)}\right. \\
+2 \sqrt{v_{S}^{+}\left(u_{i}\right) v_{C}^{+}\left(u_{i}\right)}+\sqrt{\pi_{S}^{-}\left(u_{i}\right) \pi_{C}^{-}\left(u_{i}\right)}+\sqrt{\pi_{S}^{+}\left(u_{i}\right) \pi_{C}^{+}\left(u_{i}\right)} \\
\left.+\sqrt{\left(1-v_{S}^{-}\left(u_{i}\right)\right)\left(1-v_{C}^{-}\left(u_{i}\right)\right)}+\sqrt{\left(1-v_{S}^{+}\left(u_{i}\right)\right)\left(1-v_{C}^{+}\left(u_{i}\right)\right)}\right] \\
\Rightarrow \operatorname{Sim}_{3}(R, C) \leq \operatorname{Sim}_{3}(S, C) .
\end{gathered}
$$

Similarly, $\operatorname{Sim}_{3}(R, C) \leq \operatorname{Sim}_{3}(S, C)$.

Thus, $\operatorname{Sim}_{3}(R, S)$ satisfies $\left(S_{4}\right)$. Hence $\operatorname{Sim}_{3}(R, S)$ holds all essential axioms of SM for IVIFSs.

Assuming the weights of $u_{i}$, we discuss the weighted SM for IVIFSs as follows:

$$
\begin{aligned}
\operatorname{Sim}_{3 w}(R, S)= & \frac{1}{4} \sum_{i=1}^{n} w_{i}\left[\sqrt{\mu_{R}^{-}\left(u_{i}\right) \mu_{S}^{-}\left(u_{i}\right)}+\sqrt{\mu_{R}^{+}\left(u_{i}\right) \mu_{S}^{+}\left(u_{i}\right)}+2 \sqrt{v_{R}^{-}\left(u_{i}\right) v_{S}^{-}\left(u_{i}\right)}\right. \\
& +2 \sqrt{v_{R}^{+}\left(u_{i}\right) v_{S}^{+}\left(u_{i}\right)}+\sqrt{\pi_{R}^{-}\left(u_{i}\right) \pi_{S}^{-}\left(u_{i}\right)}+\sqrt{\pi_{R}^{+}\left(u_{i}\right) \pi_{S}^{+}\left(u_{i}\right)} \\
& \left.+\sqrt{\left(1-v_{R}^{-}\left(u_{i}\right)\right)\left(1-v_{S}^{-}\left(u_{i}\right)\right)}+\sqrt{\left(1-v_{R}^{+}\left(u_{i}\right)\right)\left(1-v_{S}^{+}\left(u_{i}\right)\right)}\right] .
\end{aligned}
$$

\subsubsection{Numerical comparison}

To demonstrate the advantage of the IVIF-similarity measures, we compared the developed approach and the similarity measures that exist in literature.

Example 3.1 Let $R_{i}$ and $S_{i}$ be two IVIFSs, we evaluate the SMs between $R_{i}$ and $S_{i}$ by numerous IVIF-SMs presented in Table 3.

In Table 2, from first and second columns, we obtain that $\operatorname{Sim}_{X C_{i}}\left(R_{1}, S_{1}\right)=\operatorname{Sim}_{X C_{i}}\left(R_{2}, S_{2}\right), \quad(i=1,2), \quad \operatorname{Sim}_{P B_{1}}\left(R_{1}, S_{1}\right)=\operatorname{Sim}_{P B_{1}}\left(R_{2}, S_{2}\right)$, when $R_{1}=R_{2}, S_{1} \neq S_{2}$. Similarly, from third column and fourth columns, we find $\operatorname{Sim}_{X C_{2}}\left(R_{3}, S_{3}\right)=\operatorname{Sim}_{X C_{2}}\left(R_{4}, S_{4}\right), \quad \operatorname{Sim}_{P B_{1}}\left(R_{3}, S_{3}\right)=\operatorname{Sim}_{P B_{1}}\left(R_{4}, S_{4}\right)$, when $R_{3}=R_{4}, S_{3} \neq S_{4}$. Consequently, from first column and fourth columns, 


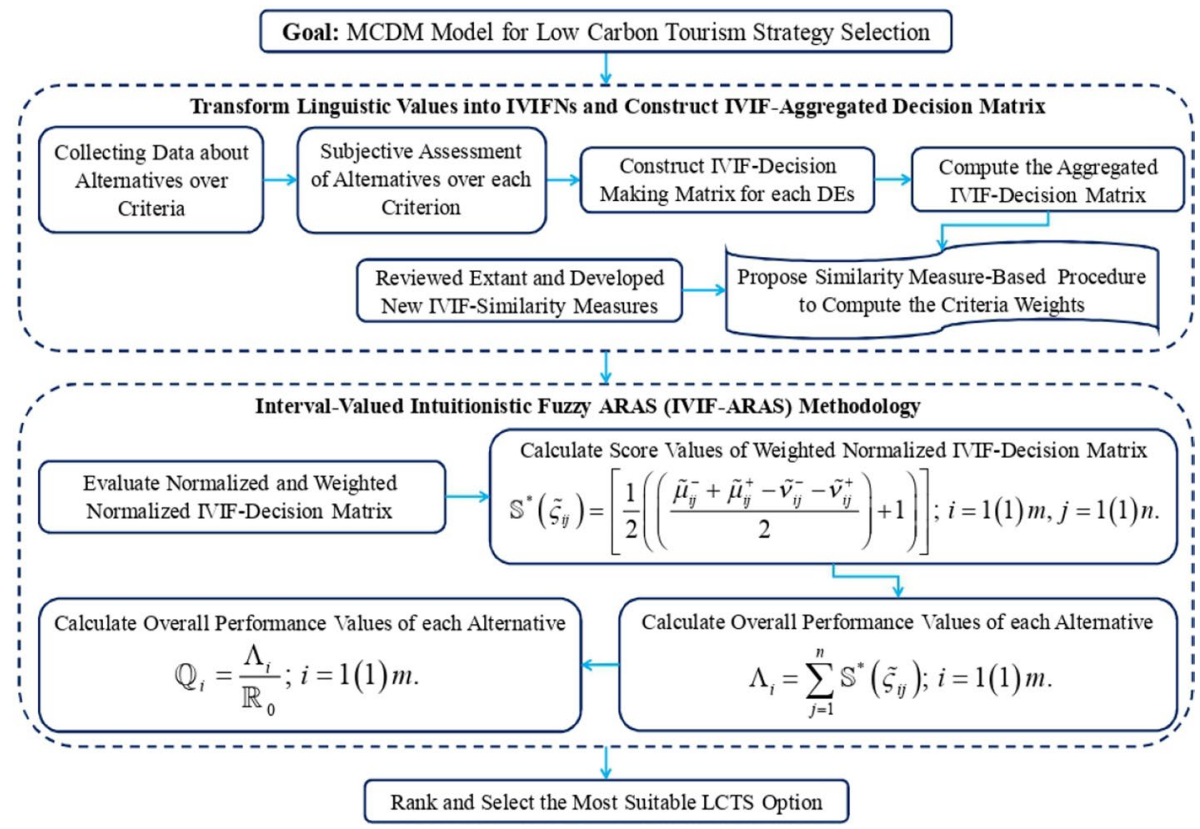

Fig. 1 Flow diagram of the proposed IVIF-ARAS method

we get that $\operatorname{Sim}_{W}\left(R_{1}, S_{1}\right)=\operatorname{Sim}_{W}\left(R_{4}, S_{4}\right), \operatorname{Sim}_{W u}\left(R_{1}, S_{1}\right)=\operatorname{Sim}_{W u}\left(R_{4}, S_{4}\right)$, $\operatorname{Sim}_{M}\left(R_{1}, S_{1}\right)=\operatorname{Sim}_{M}\left(R_{4}, S_{4}\right), \operatorname{Sim}_{M R_{2}}\left(R_{1}, S_{1}\right)=\operatorname{Sim}_{M R_{2}}\left(R_{4}, S_{4}\right)$, when $R_{1} \neq R_{4}, S_{1} \neq S_{4}$. On the similar line, from second and third columns, $S_{W}\left(R_{2}, S_{2}\right)=S_{W}\left(R_{3}, S_{3}\right)$, $\operatorname{Sim}_{W u}\left(R_{2}, S_{2}\right)=\operatorname{Sim}_{W u}\left(R_{3}, S_{3}\right), \quad \operatorname{Sim}_{M}\left(R_{2}, S_{2}\right)=\operatorname{Sim}_{M}\left(R_{3}, S_{3}\right)$, $\operatorname{Sim}_{M R_{2}}\left(R_{2}, S_{2}\right)=\operatorname{Sim}_{M R_{2}}\left(R_{3}, S_{3}\right), \operatorname{Sim}_{1}\left(R_{2}, S_{2}\right)=\operatorname{Sim}_{1}\left(R_{3}, S_{3}\right)$, when $R_{2} \neq R_{3}, S_{2} \neq S_{3}$.

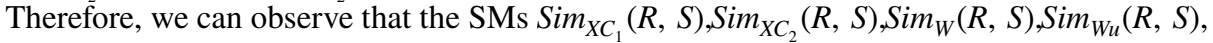
$\operatorname{Sim}_{M}(R, S), \operatorname{Sim}_{P B_{1}}(R, S), \operatorname{Sim}_{M R_{2}}(R, S)$ and $\operatorname{Sim}_{1}(R, S)$ are not reasonable. Therefore, we conclude that the proposed IVIF-SMs $\operatorname{Sim}_{Y_{2}}(R, S), \operatorname{Sim}_{2}(R, S)$ and $\operatorname{Sim}_{3}(R, S)$ overcome these shortcomings. As a result, novel IVIF-SMs are more reasonable than existing ones.

Example

3.2 Let

$S=\{\langle u,[0.3,0.4],[0.3,0.5]\rangle: u \in U\}$.

$$
R=\{\langle u,[0.2,0.3],[0.4,0.6]\rangle: u \in U\}
$$

and $T=\{\langle u,[0.3,0.4],[0.4,0.6]\rangle: u \in U\}$ be three IVIFSs.

For each $u \in U$, the NG of $R$ and $T$ is same, but the change of the BG of $R$ and $T$ is from $[0.2,0.3]$ into [0.3, 0.4], while for each $u \in U$, the NG between $R$ and $S$ is different from $[0.4,0.6]$ into $[0.3,0.5]$ and the BG is the same as that of $R$ and $T$. Consequently, $R$ is more similar to $T$ than to $S$. We now evaluate $\operatorname{Sim}_{1}(R, S), \operatorname{Sim}_{X C_{1}}^{\infty}(R, S)$ and $\operatorname{Sim}_{X C_{2}}^{1}(R, S)$. Then, $\operatorname{Sim}_{1}(R, S)=0.8182$ and $\operatorname{Sim}_{1}(R, T)=0.9091$, which point out that IVIFS $R$ is more similar to $T$ than to $S$, which is consistent with intuition.

Next, we calculate the $\operatorname{SMs} \operatorname{Sim}_{X C_{1}}^{\infty}(R, S)$ and $\operatorname{Sim}_{X C_{2}}^{1}(R, S)$; then $\operatorname{Sim}_{X C_{1}}^{\infty}(R, S)=\operatorname{Sim}_{X C_{1}}^{\infty}(R, T)=\operatorname{Sim}_{X C_{2}}^{1}(R, S)=\operatorname{Sim}_{X C_{2}}^{1}(R, T)=0.9$, which are not reasona- 
ble. Therefore, the SMs $\operatorname{Sim}_{1}(R, S)$, Eq. (8), Eq. (11) and Eq. (12) are demonstrated to be more reasonable than $\operatorname{Sim}_{X C_{1}}^{\infty}(R, S)$ and $\operatorname{Sim}_{X C_{2}}^{1}(R, S)$ in some cases.

\subsection{Proposed interval-valued intuitionistic fuzzy ARAS approach}

The process of decision making comprises a logical and systematic way to choose a feasible one with multiple alternatives. In real-life decision-making problems, some problems consider only a single criterion for each one of the alternatives, and the problem is concentrated on single-criterion decision making which is simpler because the outcome is obtained completely by choosing the one with premium single criterion, while many concerns are assessed over various criteria. It revolves such problems into MCDM procedures, where numerous MCDM methods implement the significance (weights) of coefficients. This study is mainly aimed at the implementation of IVIF-ARAS method in a way to select the best low-carbon tourism strategy (LCTS). Here, the criteria weights for LCTS are computed by proposed IVIF similarity measure. When the weights are calculated, IVIF-ARAS approach is implemented to determine the desirable LCTS. IVIF-ARAS method implements logical comparison in the ratio of the sum to estimate the weights and normalization of the attribute values in order to obtain the utility degree of the alternative in IVIFSs. Figure 1 displays the workflow of the developed method and is briefly described as follows:

Step 1: The formulation of the alternative and criteria.

The main aim of the MCDM process is choosing the best options from a set of $m$ options $M=\left\{M_{1}, M_{2}, \ldots, M_{m}\right\}$ under the set of criteria $S=\left\{S_{1}, S_{2}, \ldots, S_{n}\right\}$. Thus, a group of $\ell$ DEs $E=\left\{E_{1}, E_{2}, \ldots, E_{\ell}\right\}$ should be constructed to reach the best alternative(s).

Step 2: The construction of the decision matrix for DEs and aggregated IVIF-decision matrix (AIVIF-DM).

Let $\mathbb{Z}=\left(\xi_{i j}^{(k)}\right)_{m \times n}$ be DEs assessment matrices and $\lambda=\left(\lambda_{1}, \lambda_{2}, \ldots, \lambda_{\ell}\right)^{T}$ be the DEs weight, where $\sum_{k=1}^{\ell} \lambda_{k}=1, \lambda_{k} \in[0,1]$. Therefore, the AIVIF-DM $\hat{\mathbb{Z}}=\left(\hat{\xi}_{i j}\right)_{m \times n}$ is computed as follows:

$$
\bar{\xi}_{i j}=\left(\left(\xi_{i j}^{(1)} \oplus \xi_{i j}^{(2)} \oplus \ldots \oplus \xi_{i j}^{(\ell)}\right) / \ell\right) ; i=1(1) m, j=1(1) n .
$$

where $\hat{\xi}_{i j}$ is an IVIFN.

Step 3: The calculation of the weight vector of criteria.

In a decision-making problem, all criteria are not having equal importance. Let $w=\left(w_{1}, w_{2}, \ldots, w_{n}\right)^{T}$ such that $\sum_{j=1}^{n} w_{j}=1, w_{j} \in[0,1]$ be a criterion weight vector. Successively to achieve $w$, we apply the following procedure:

$$
w_{j}=\frac{\sum_{i=1}^{m} \sum_{t=1, t \neq i}^{m}\left(1-\operatorname{Sim}\left(\hat{\xi}_{i j}, \hat{\xi}_{i j}\right)\right)}{\sum_{j=1}^{n} \sum_{i=1}^{m} \sum_{t=1, t \neq i}^{m}\left(1-\operatorname{Sim}\left(\hat{\xi}_{i j}, \hat{\xi}_{i j}\right)\right)}, j=1(1) n .
$$

Step 4: Determine optimal performance rating 


$$
\mathbb{R}_{0}= \begin{cases}\max \hat{\xi}_{i j}, j \in S_{b} \\ \min \hat{\xi}_{i j}, j \in S_{n}\end{cases}
$$

where $S_{b}$ and $S_{n}$ stand for the benefit-type and cost-type attributes, respectively.

Step 5: The generation of normalized AIVIF-DM.

All of the individual decision judgments involved need to be integrated into a group judgment in order to construct normalized AIVIF-DM $\mathbb{N}=\left(\varsigma_{i j}\right)_{m \times n}$ such that

$$
\varsigma_{i j}=\left\{\begin{array}{ll}
\left\langle\left[\mu_{i j}^{-}, \mu_{i j}^{+}\right],\left[v_{i j}^{-}, v_{i j}^{+}\right]\right\rangle, & j \in S_{b} \\
\left\langle\left[v_{i j}^{-}, v_{i j}^{+}\right],\left[\mu_{i j}^{-}, \mu_{i j}^{+}\right]\right\rangle, & j \in S_{n}
\end{array} ; i=1(1) m,\right.
$$

Step 6: The construction of weighted normalized AIVIF-DM (WNAIVIF-DM).

When the weight vector $w=\left(w_{1}, w_{2}, \ldots, w_{n}\right)^{T}$ of the criteria $S_{j}: j=1(1) n$ is obtained, the WNAIVIF-DM $\mathbb{N}_{w}=\left(\tilde{\zeta}_{i j}\right)_{m \times n}$ is created as follows:

$$
\tilde{\varsigma}_{i j}=\bigoplus_{j=1}^{n} w_{j} \varsigma_{i j}=\left\langle\left[1-\prod_{j=1}^{n}\left(1-\mu_{i j}^{-}\right)^{w_{j}}, 1-\prod_{j=1}^{n}\left(1-\mu_{i j}^{+}\right)^{w_{j}}\right],\left[\prod_{j=1}^{n}\left(v_{i j}^{-}\right)^{w_{j}}, \prod_{j=1}^{n}\left(v_{i j}^{+}\right)^{w_{j}}\right]\right\rangle,
$$

where $\tilde{\varsigma}_{i j}=\left\langle\left[\tilde{\mu}_{i j}^{-}, \tilde{\mu}_{i j}^{+}\right],\left[\tilde{v}_{i j}^{-}, \tilde{v}_{i j}^{+}\right]\right\rangle$is the weighted IVIFN.

Step 7: Scoring the values of WNAIVIF-DM.

By Eq. (4), the score values of WNAIVIF-DM $\mathbb{N}_{w}=\left(\tilde{\zeta}_{i j}\right)_{m \times n}$ are computed by

$$
\mathbb{S}^{*}\left(\tilde{\varsigma}_{i j}\right)=\left[\frac{1}{2}\left(\left(\frac{\tilde{\mu}_{i j}^{-}+\tilde{\mu}_{i j}^{+}-\tilde{v}_{i j}^{-}-\tilde{v}_{i j}^{+}}{2}\right)+1\right)\right] ; i=1(1) m, j=1(1) n .
$$

Step 8: The computation of general performance value and degree of utility.

The general performance rating of each alternative is evaluated using the following equation:

$$
\Lambda_{i}=\sum_{j=1}^{n} \mathbb{S}^{*}\left(\tilde{\zeta}_{i j}\right), \quad i=1(1) m .
$$

The largest value is measured as the best, while the smallest value is the worst. According to the process of evaluation, the best function $\Lambda_{i}$ is directly and proportionally associated with $\xi_{i j}$ and weights $w_{j}$ of the examined criteria and their relative effect upon the final results. Thus, the bigger value of $\Lambda_{i}$, the more efficient the alternative. The alternatives priority is evaluated based on the value of $\Lambda_{i}$. As a result, this is appropriate to estimate and rank the alternatives once the proposed method is implemented.

For estimating the alternatives, it is significant not only to assess the optimal alternative but also to assess the comparative performance of given alternatives regarding the optimal one. As a result, the degree of variant utility is calculated using comparison method and the examined variant with the ideally optimal one $\mathbb{R}_{0}$. The utility degree $\mathbb{Q}_{i}$ of each option $M_{i}: i=1(1) m$ is computed by 
Table 4 Detail description of the selected criteria for evaluation of LCTS selection (Zhang, 2017)

\begin{tabular}{lll}
\hline Dimension & Criteria & Type \\
\hline Social & Education of low-carbon environment $\left(S_{1}\right)$ & Benefit \\
& Carbon literacy of residents $\left(S_{2}\right)$ & Benefit \\
& Carbon literacy of tourists $\left(S_{3}\right)$ & Benefit \\
& Special plans for low-carbon tourism $\left(S_{4}\right)$ & Benefit \\
Economy & Proportion of low-carbon tourist $\left(S_{5}\right)$ & Benefit \\
& Proportion of green hotel $\left(S_{6}\right)$ & Benefit \\
& Proportion of green catering enterprise $\left(S_{7}\right)$ & Benefit \\
& Low-carbon transportation $\left(S_{8}\right)$ & Benefit \\
& Tourism carbon intensity $\left(S_{9}\right)$ & Cost \\
Environmental & Air pollution index $\left(S_{10}\right)$ & Cost \\
& Noise pollution level $\left(\mathrm{S}_{11}\right)$ & Cost \\
& Environmental protection $\left(S_{12}\right)$ & Benefit \\
\hline
\end{tabular}

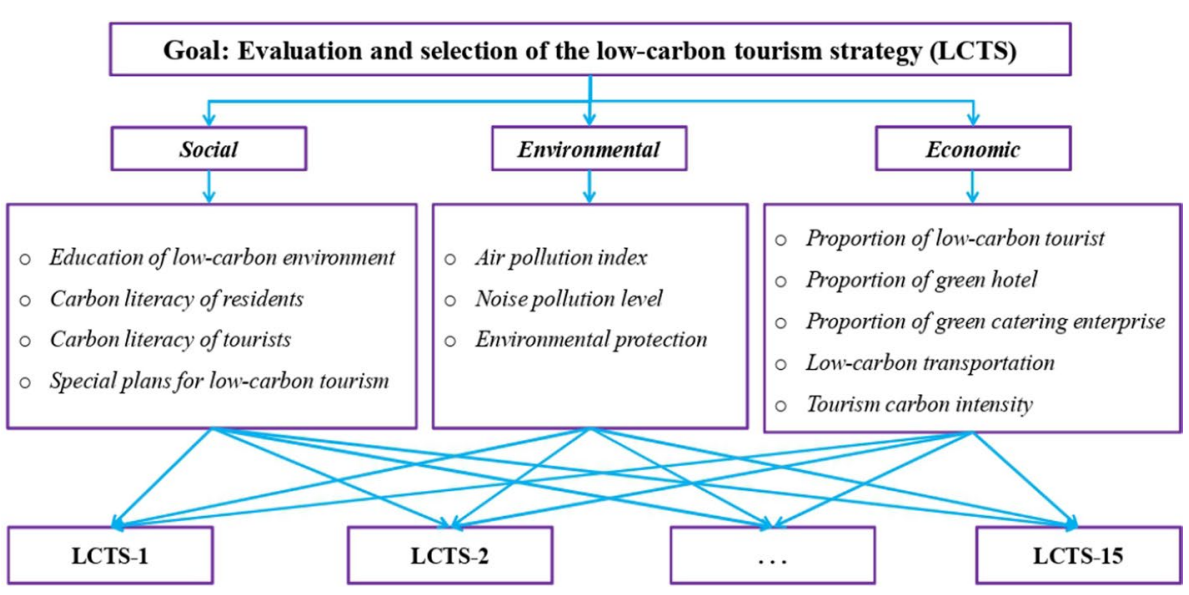

Fig. 2 Hierarchical structure of selecting criteria for LCTS selection

$$
\mathbb{Q}_{i}=\frac{\Lambda_{i}}{\mathbb{R}_{0}} ; i=1(1) m
$$

It is observed that $\mathbb{Q}_{i} \in[0,1]$ and it could be structured in an increasing sequence. According to the utility function, the relative efficiency of a feasible option can be evaluated.

Step 9: The selection of the most desirable one.

The given options are graded in ascending order $\mathbb{Q}_{i}$, i.e., the option with the maximum degree of $\mathbb{Q}_{i}$ is the optimal one. Hence, the best alternative could be specified by

$$
M^{*}=\left\{M_{i} \mid \max _{i} \mathbb{Q}_{i} ; i=1(1) m\right\},
$$

where $M^{*}$ is the optimal alternative, $i=1(1) m$. 
Table 5 The scale for the criteria rating and the LCTS options based on LVs

\begin{tabular}{ll}
\hline LVs & IVIFNs \\
\hline Extremely low (EL)/extremely bad (EB) & $\langle[0.05,0.10],[0.85,0.90]\rangle$ \\
Very low (VL)/very bad (VB) & $\langle[0.10,0.20],[0.70,0.75]\rangle$ \\
Low (L)/bad (B) & $\langle[0.20,0.30],[0.55,0.65]\rangle$ \\
Medium low (ML)/medium bad (MB) & $\langle[0.30,0.40],[0.45,0.55]\rangle$ \\
Medium (M)/fair (F) & $\langle[0.45,0.55],[0.35,0.40]\rangle$ \\
Medium high $(\mathrm{MH}) /$ medium good (MG) & $\langle[0.55,0.65],[0.25,0.30]\rangle$ \\
High $(\mathrm{H}) /$ good $(\mathrm{G})$ & $\langle[0.65,0.75],[0.15,0.20]\rangle$ \\
Very high $(\mathrm{VH}) /$ very good (VG) & $\langle[0.75,0.90],[0.05,0.10]\rangle$ \\
Extremely high $($ EH)/extremely good (EG) & $\langle[0.90,1.00],[0.00,0.00]\rangle$ \\
\hline
\end{tabular}

\section{Results and discussion}

\subsection{Case study: low-carbon tourism strategy (LCTS) selection}

In this study, an approach containing ARAS and SMs for IVIFSs is applied to explain the LCTS selection problem.

In order to define LCTSs, we create a group of DEs. They constructed some strategies that have been executed in other LCT destinations in India. Then, we have studied the related literature to articulate option LCTSs for India. Lastly, we have determined 15 LCT development strategies, which are denoted as $M_{1}, M_{2}, \ldots, M_{15}$. Though every strategy for carbon emission reduction is a decent proposal, it is essential to rank each of the different strategies so that they can be applied in a systematic way. Commonly, economic, environmental and social aspects are taken and revealed in the criteria for any assessment of LCTSs. In this problem, these LCTSs are assessed over the considered 12 criteria and are specified in Table 4 and Fig. 2.

Here, Table 5 depicts the linguistic values (LVs) in term of IVIFNs for the criteria and the LCTS options. Table 6 shows the LVs $\left(E_{1}, E_{2}, E_{3}\right)$ based on DEs for the criteria of considered LCTS options.

Using Eq. (25), the AIVIF-DM for LCTSs is computed using the DEs' opinions and mentioned in Table 7. Corresponding to Table 7 and Eq. (26), the criterion weight based on a SM Eq. (13) is evaluated by

$$
w_{j}=\{0.0768,0.0377,0.0826,0.0693,0.0523,0.0818,0.0732,0.1886,0.0715,0.1533,0.0421,0.0708\} .
$$

The first step in using the proposed IVIF-ARAS approach is the determination of the optimum performance degree $\left(\mathbb{R}_{0}\right)$ of LCTS options, and this is done by using Eq. (27) and is presented in Table 8 .

Using Eq. (28), the normalized AIVIF-DM is evaluated in Table 8, and the WNAIVIFDM for LCTS options is formed based on Table 9 and Eq. (29) and given in Table 10.

Using Table 10 and Eq. (30), the score values $\mathbb{S}^{*}\left(\tilde{\zeta}_{i j}\right)$ of IVIFNs are given in Table 11. Based on Eqs. (31) and (32), overall performance rating $\left(\Lambda_{i}\right)$ and degree of utility or relative quality $\left(\mathbb{Q}_{i}\right)$ of each LCTS option are computed and demonstrated in Table 10. Then, the ranking order of LCTSs is determined as $M_{11}>M_{4}>M_{10}>M_{14}>M_{5}>M_{7}>M_{9}>M_{13}$ $>M_{2}>M_{6}>M_{12}>M_{8}>M_{15}>M_{1}>M_{3}$. Hence, the desirable LCTS alternative is $M_{11}$. 


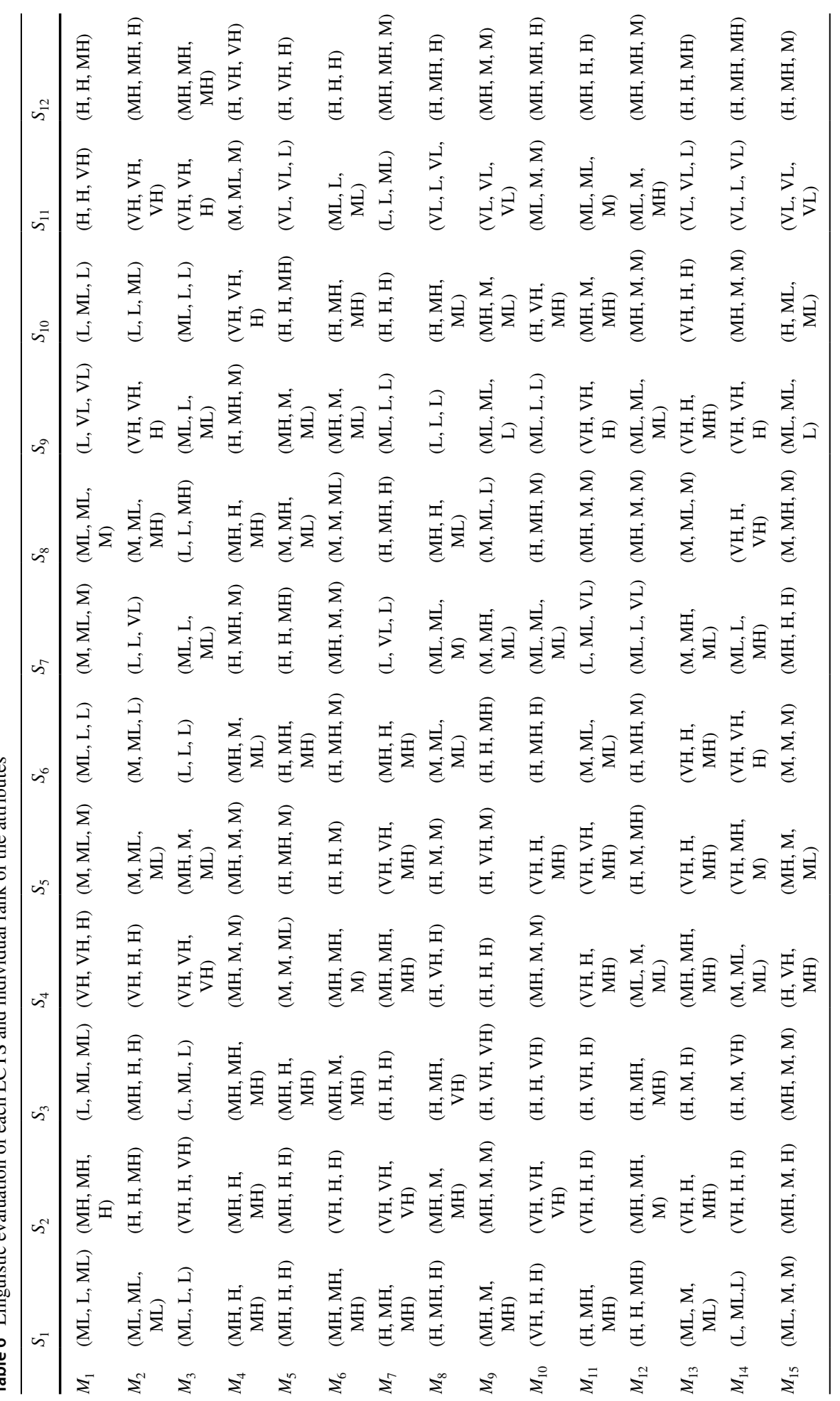




\subsection{Comparative study}

A comparison is presented for the purpose of validating the outcomes of IVIF-ARAS methodology with IVIF-TOPSIS model. To facilitate the comparative study, we prefer the IVIF-TOPSIS method proposed by Bai (2013) with the above MCDM problem given by.

The TOPSIS model begins with the evaluation of the IVIF-ideal solution $\xi^{+}$(IVIF-IS) and the IVIF-anti-ideal solution $\xi^{-}$(IVIF-AIS). Let $S_{b}$ and $S_{n}$ be the sets benefit-type and cost-type criteria, respectively. According to IVIFSs doctrine and the conventional TOPSIS model, $\xi^{+}$and $\xi^{-}$are given by

$$
\begin{aligned}
\xi^{+}= & \left\{M_{i},\left\langle\max _{i}\left[\mu_{i j}^{-}, \mu_{i j}^{+}\right]\left|j \in S_{b}, \min _{i}\left[\mu_{i j}^{-}, \mu_{i j}^{+}\right]\right| j \in S_{n}\right\rangle,\right. \\
& \left.\left.\left\langle\min _{i}\left[v_{i j}^{-}, v_{i j}^{+}\right]\left|j \in S_{b}, \max _{i}\left[v_{i j}^{-}, v_{i j}^{+}\right]\right| j \in S_{n}\right\rangle\right): i=1,2, \ldots, m\right\}, \\
\xi^{-}= & \left\{\left(M_{i},\left\langle\min _{i}\left[\mu_{i j}^{-}, \mu_{i j}^{+}\right]\left|j \in S_{b}, \max _{i}\left[\mu_{i j}^{-}, \mu_{i j}^{+}\right]\right| j \in S_{n}\right\rangle,\right.\right. \\
& \left.\left.\left\langle\max _{i}\left[v_{i j}^{-}, v_{i j}^{+}\right]\left|j \in S_{b}, \min _{i}\left[v_{i j}^{-}, v_{i j}^{+}\right]\right| j \in S_{n}\right\rangle\right): i=1,2, \ldots, m\right\} .
\end{aligned}
$$

Thus, the corresponding IVIF-IS and IVIF-AIS in the above decision-making problem can be obtained by Eqs. $(34,35)$, mentioned as follows:

$\xi^{+}=\{([0.6871,0.8158],[0.1040,0.1587]),([0.7500,0.9000],[0.0500,0.1000])$, ([0.7203, 0.8643], [0.0721,0.1260]), ([0.7500, 0.9000], [0.0500, 0.1000], ([0.6959, 0.8482], $[0.0855,0.1442]),([0.7203,0.8643],[0.0721,0.1260]),([0.6194,0.7203],[0.1778,0.2289])$, $([0.7203,0.8643],[0.0721,0.1260]),([0.1347,0.2348],[0.6459,0.7151]),([0.6871$, $0.8158],[0.1040,0.1587]),([0.1000,0.2000],[0.7000,0.7500]),([0.7203,0.8643],[0.0721,0.1260])\}$, $\xi^{-}=\{([0.2348,0.3351],[0.5144,0.6148]),([0.4856,0.5862],[0.3129,0.3634]),([0.2348$, $0.3351],[0.5144,0.6148]),([0.3541,0.4549],[0.4138,0.4946]),([0.3541,0.4549],[0.4138$, $0.4946]),([0.2348,0.3351],[0.5144,0.6148]),([0.1680,0.2681],[0.5960,0.6818]),([0.3396$, $0.4449],[0.4229,0.5023]),([0.7203,0.8643],[0.0721,0.1260]),([0.2348,0.3351],[0.5144$, 0.6148]), ([0.7500, 0.9000], [0.0500, 0.1000]), ([0.1500, 0.2000], [0.5189, 0.6194])\}.

Based on Eq. (24), a weighted similarity measure between the alternatives, and IVIF-IS and IVIF-AIS are given by

$$
\begin{aligned}
& \operatorname{Sim}_{3 w}\left(M_{i}, \xi^{+}\right)=\frac{1}{4} \sum_{i=1}^{n} w_{i}\left[\sqrt{\mu_{\mathbb{M}_{i}}^{-}\left(u_{i}\right) \mu_{\xi^{+}}^{-}\left(u_{i}\right)}+\sqrt{\mu_{\mathbb{M}_{i}}^{+}\left(u_{i}\right) \mu_{\xi^{+}}^{+}\left(u_{i}\right)}+2 \sqrt{v_{\mathbb{M}_{i}}^{-}\left(u_{i}\right) v_{\xi^{+}}^{-}\left(u_{i}\right)}\right. \\
& +2 \sqrt{v_{\mathbb{M}_{i}}^{+}\left(u_{i}\right) v_{\xi^{+}}^{+}\left(u_{i}\right)}+\sqrt{\pi_{\mathbb{M}_{i}}^{-}\left(u_{i}\right) \pi_{\xi^{+}}^{-}\left(u_{i}\right)}+\sqrt{\pi_{\mathbb{M}_{i}}^{+}\left(u_{i}\right) \pi_{\xi^{+}}^{+}\left(u_{i}\right)} \\
& \left.+\sqrt{\left(1-v_{\mathbb{M}_{i}}^{-}\left(u_{i}\right)\right)\left(1-v_{\xi^{+}}^{-}\left(u_{i}\right)\right)}+\sqrt{\left(1-v_{\mathbb{M}_{i}}^{+}\left(u_{i}\right)\right)\left(1-v_{\xi^{+}}^{+}\left(u_{i}\right)\right)}\right]
\end{aligned}
$$




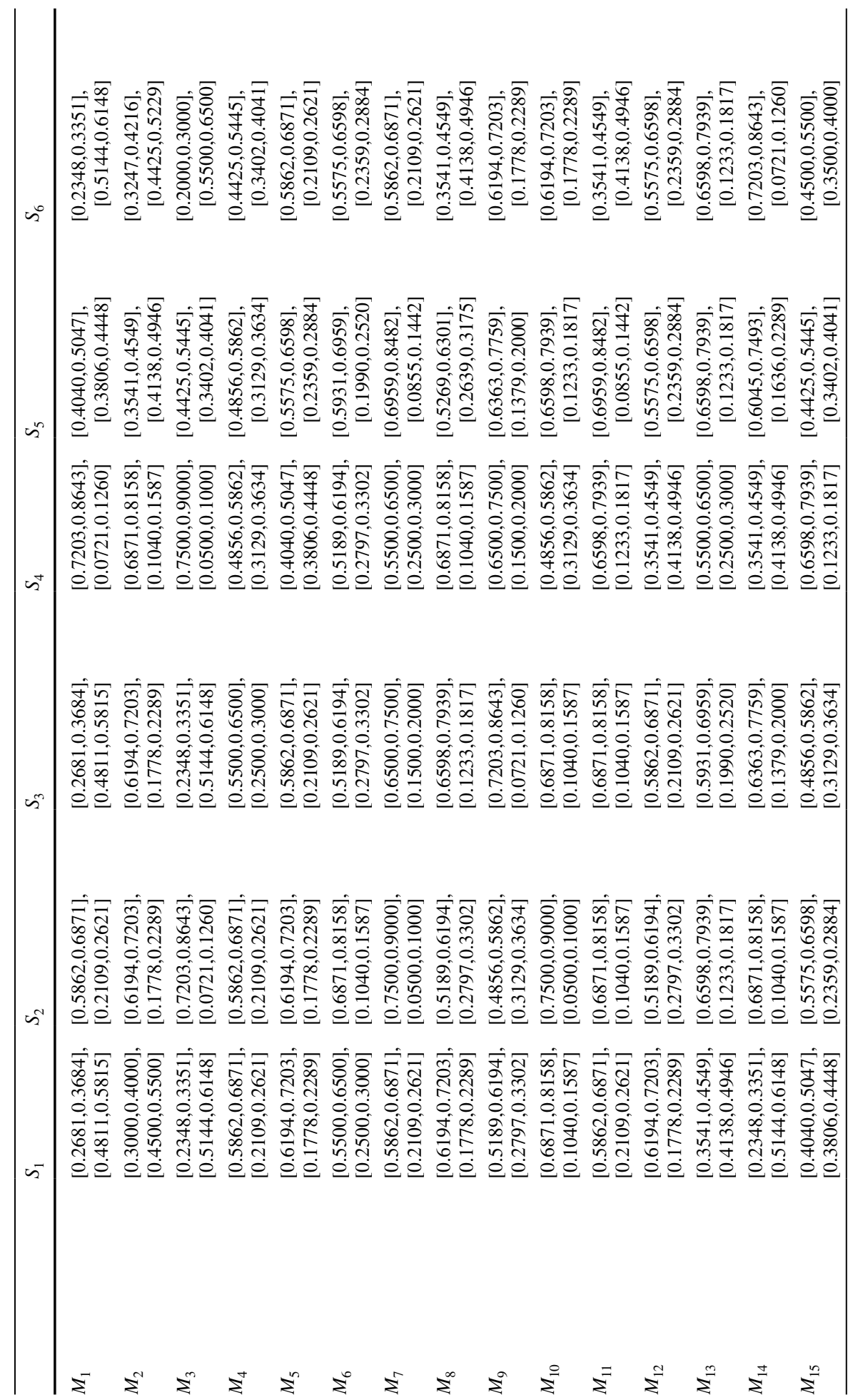




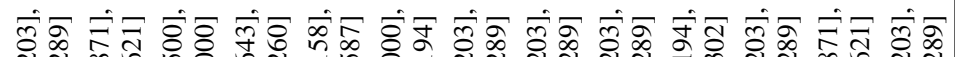

궁

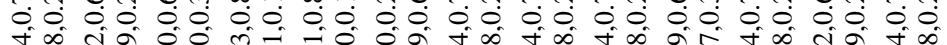
ปิ

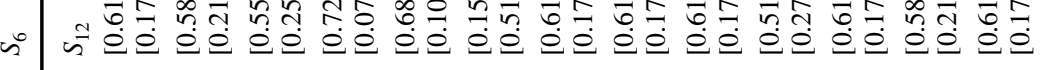

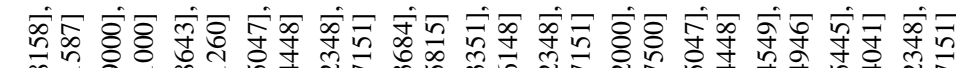

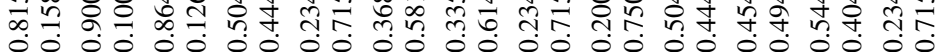
O.

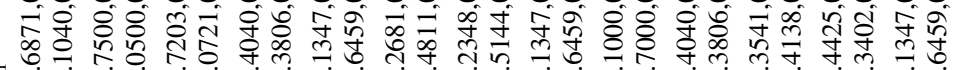

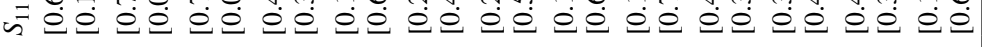

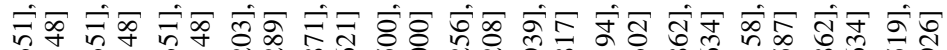
no 0 0 क オ

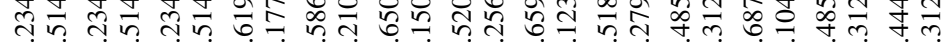

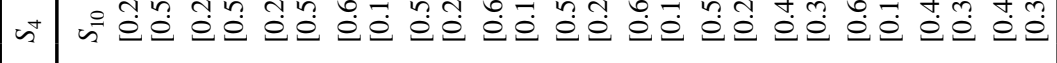

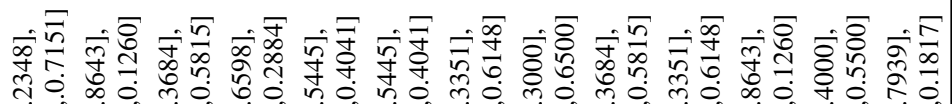
○ी

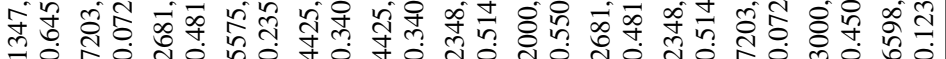

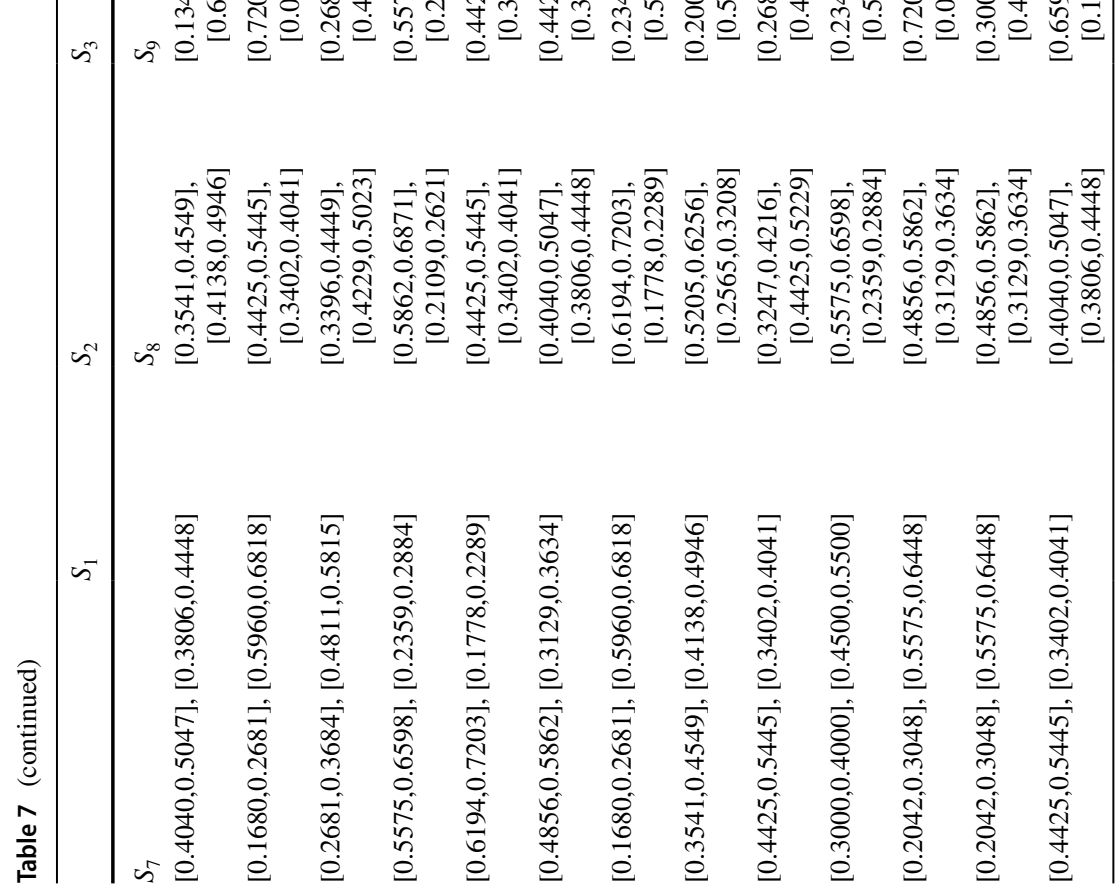




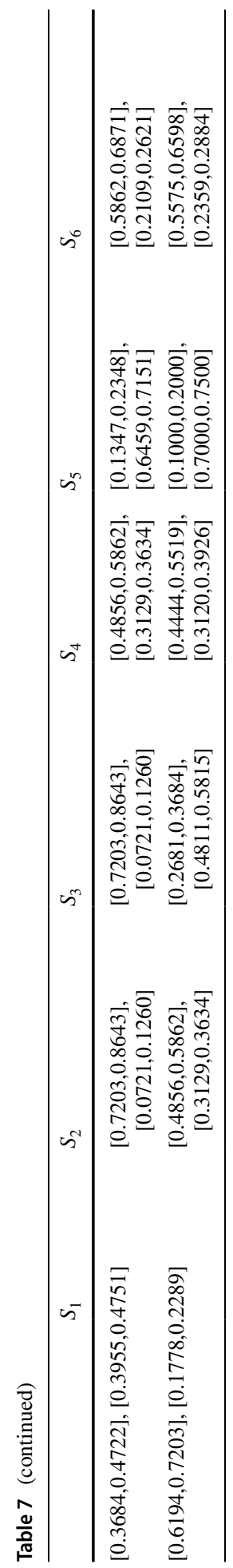




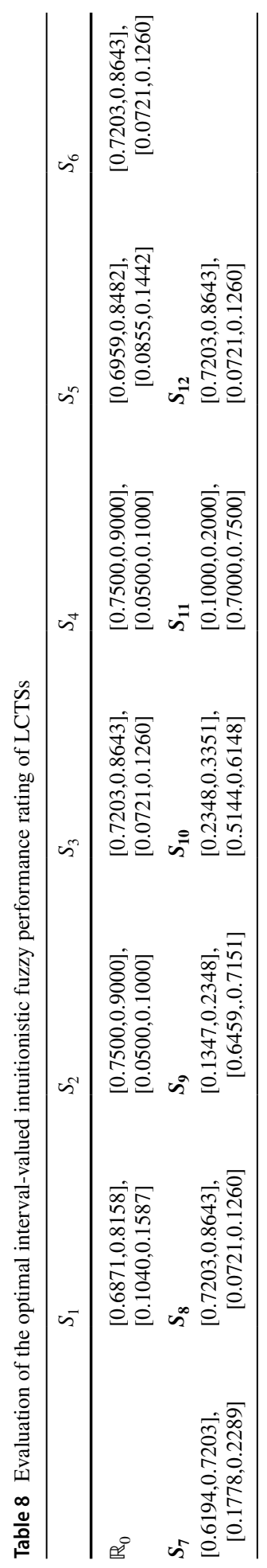




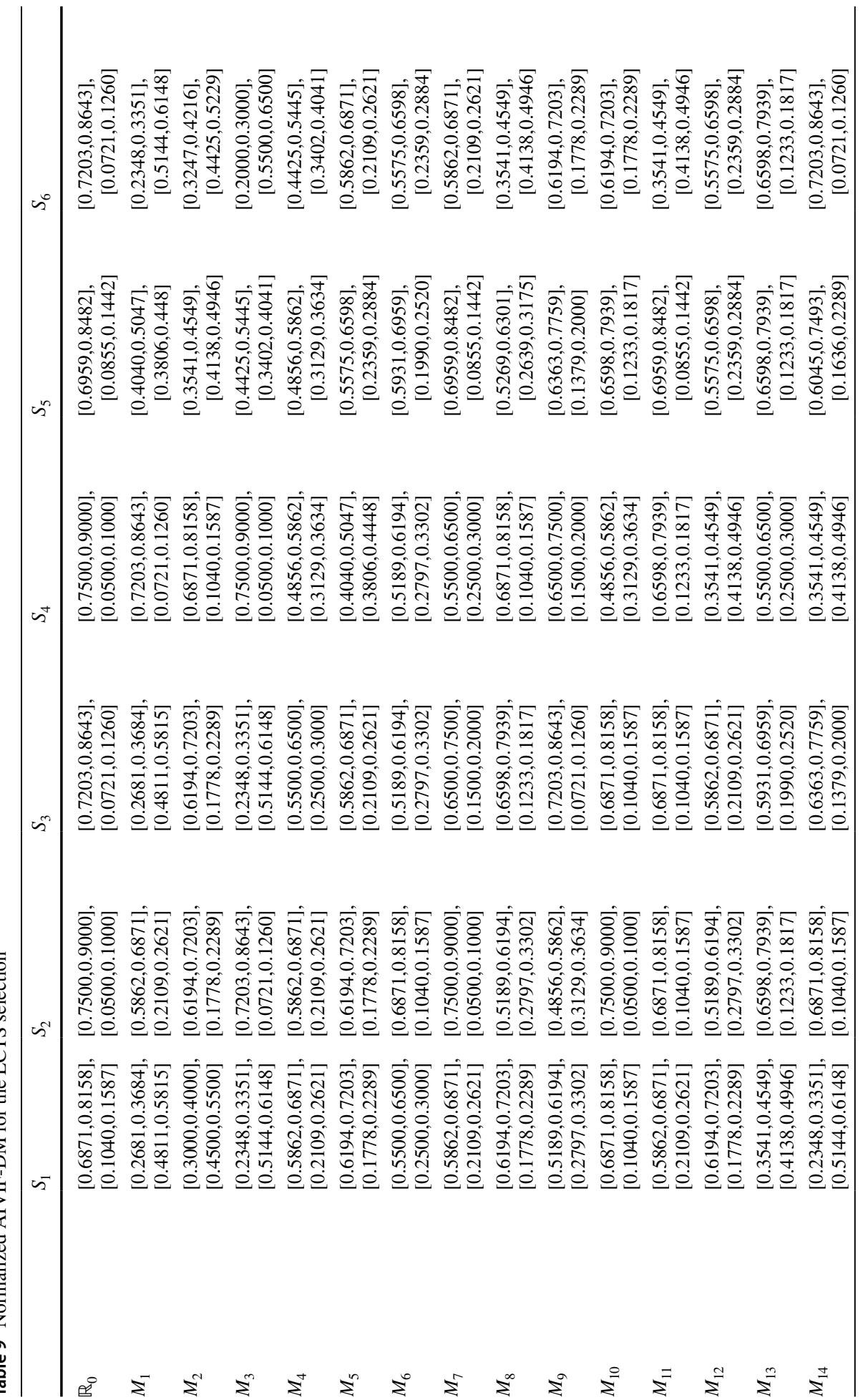




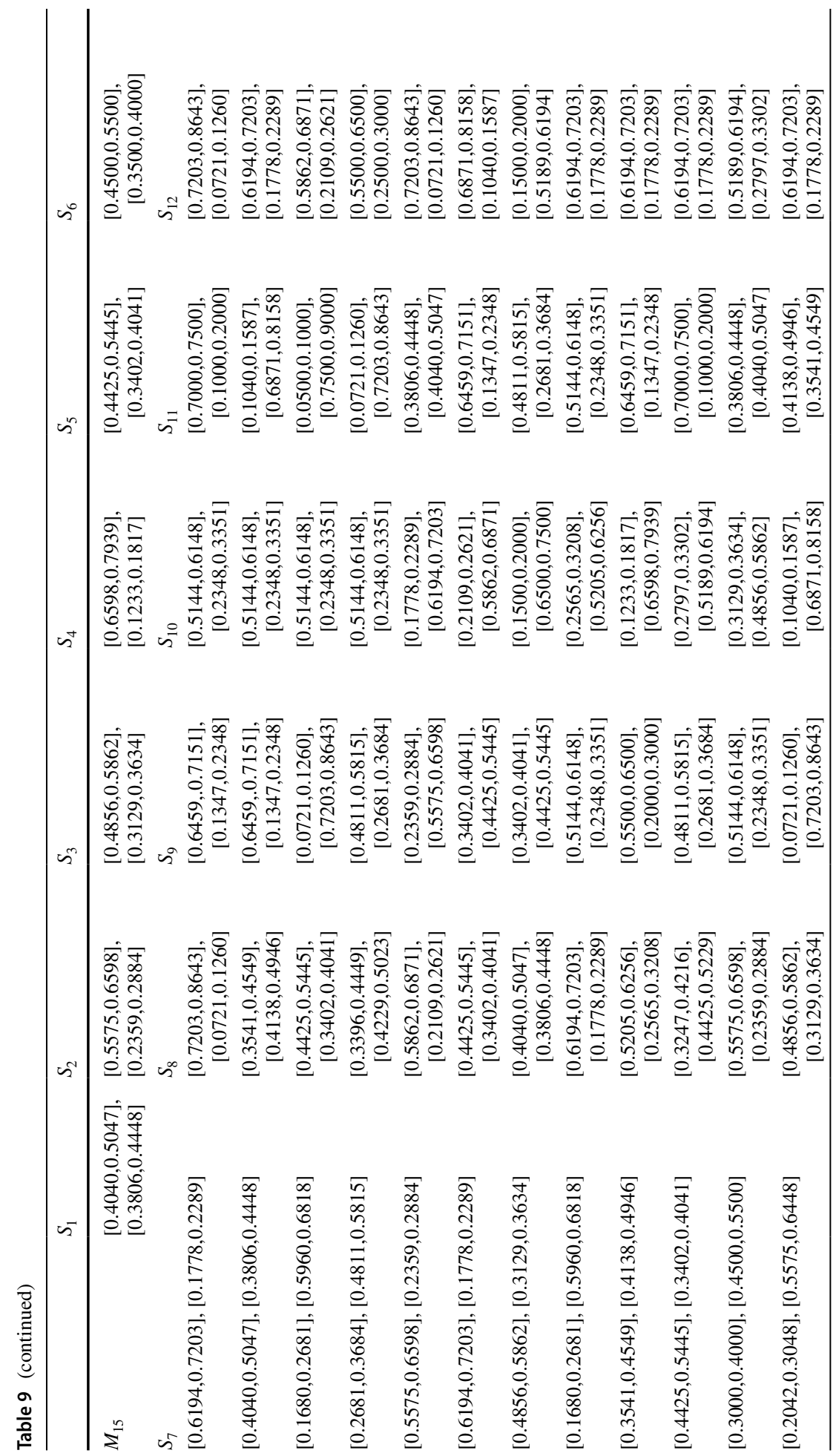




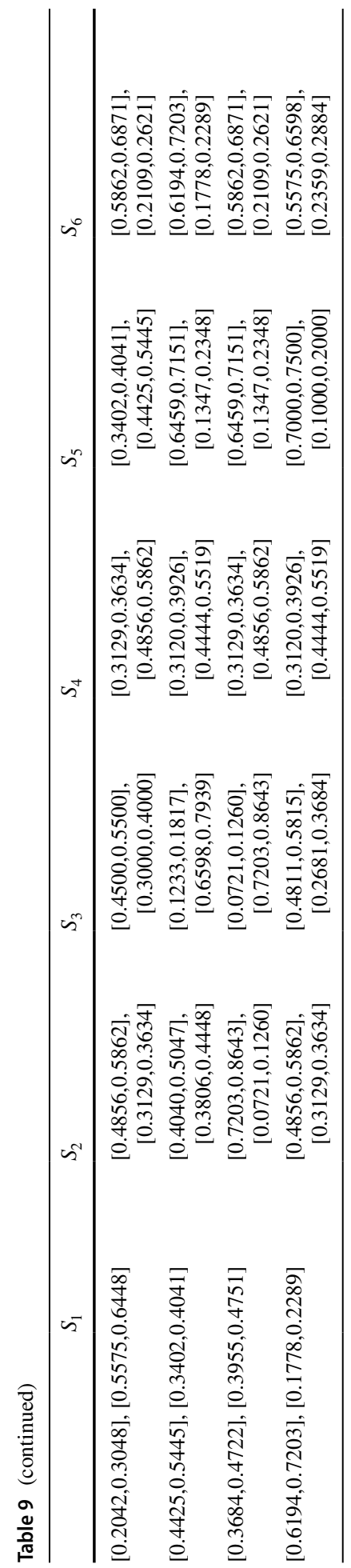




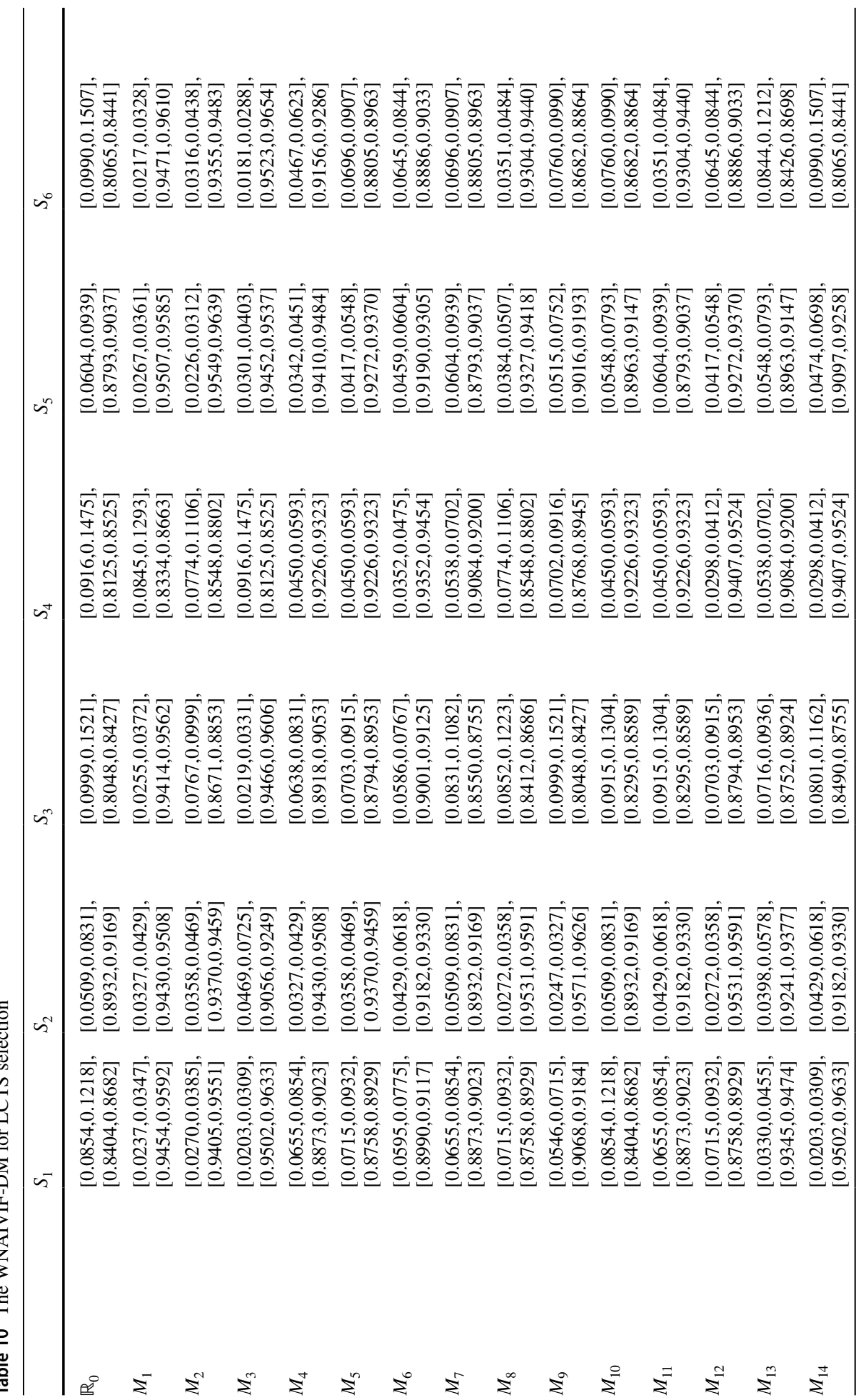




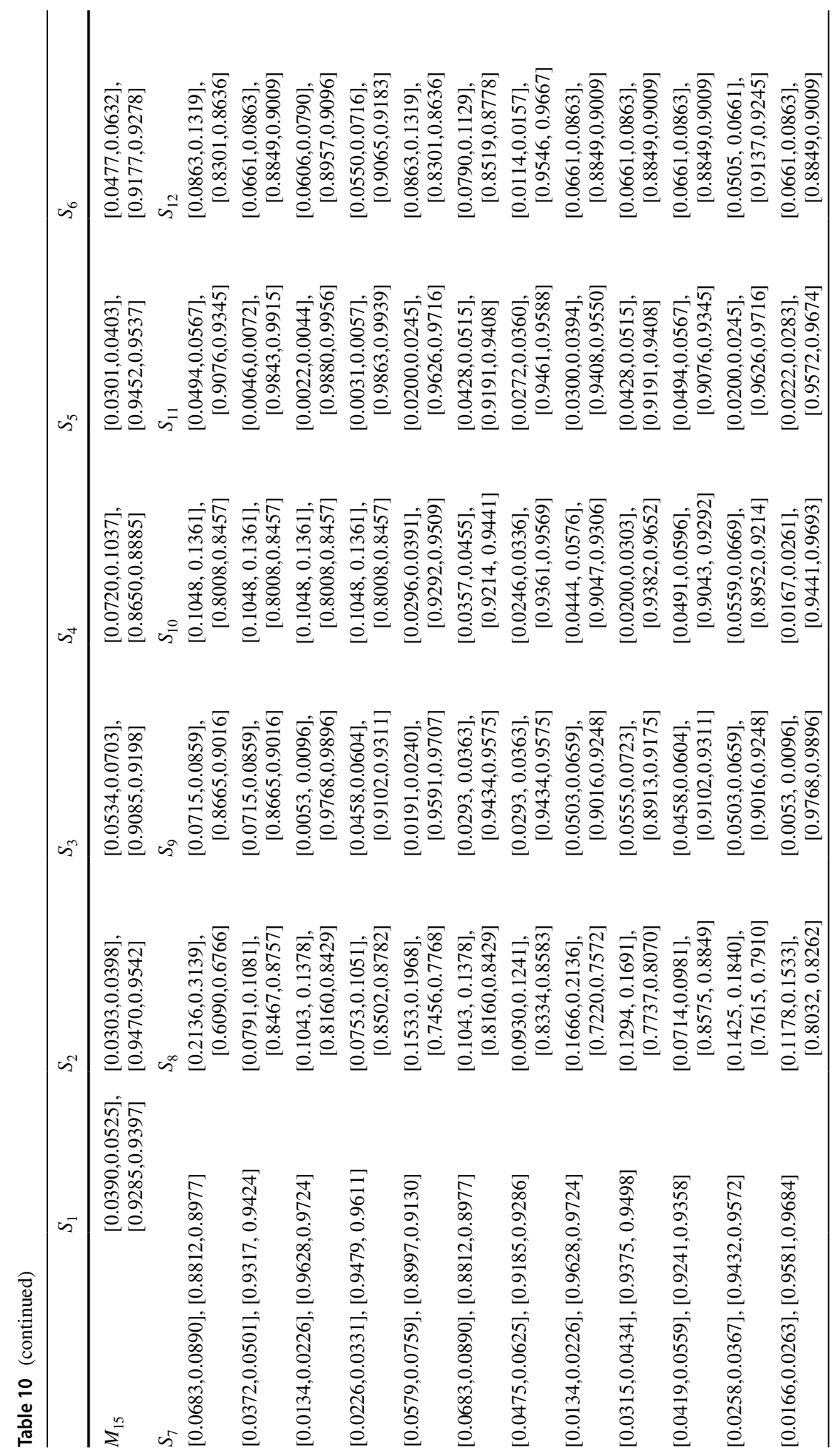




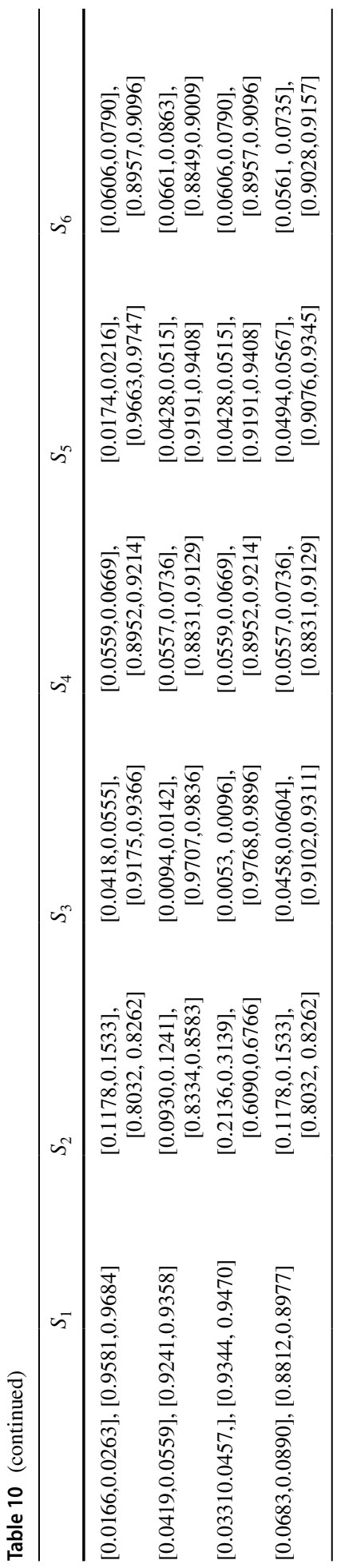




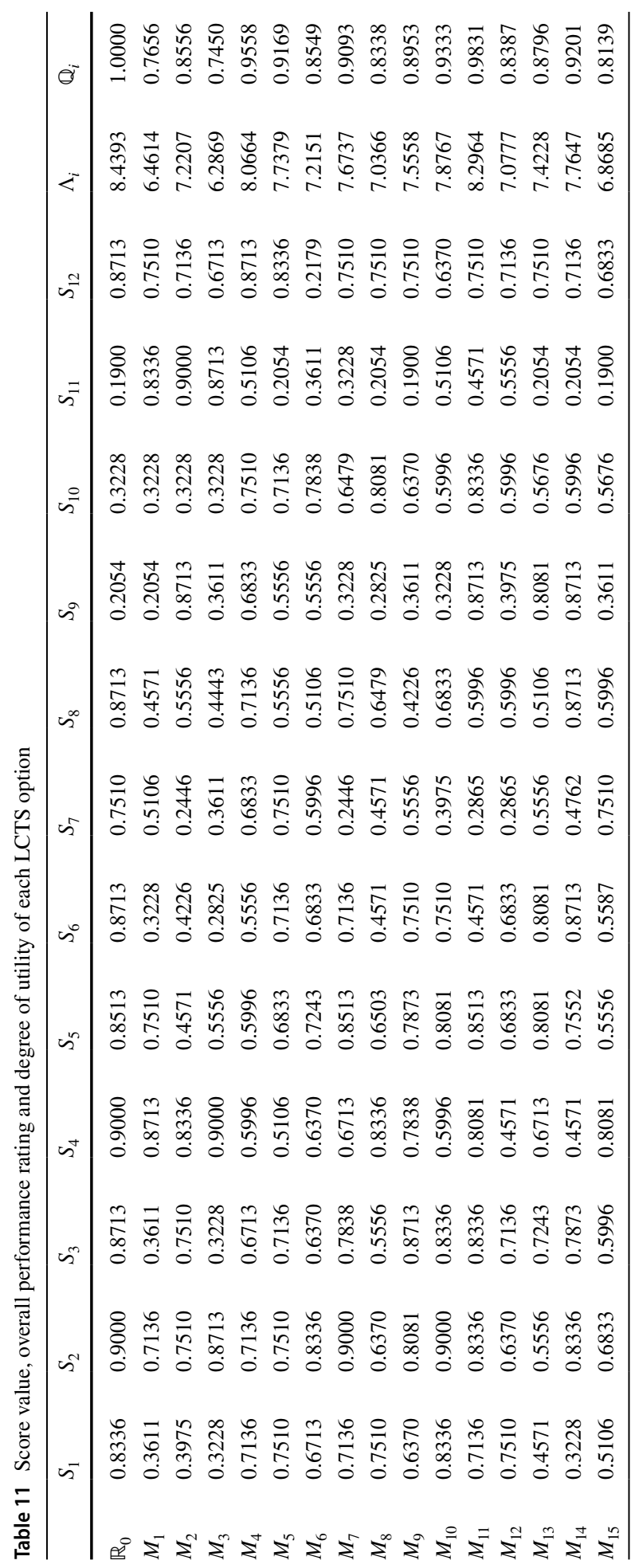




$$
\begin{aligned}
& \operatorname{Sim}_{3 w}\left(M_{i}, \xi^{-}\right)=\frac{1}{4} \sum_{i=1}^{n} w_{i}\left[\sqrt{\mu_{\mathbb{M}_{i}}^{-}\left(u_{i}\right) \mu_{\xi^{-}}^{-}\left(u_{i}\right)}+\sqrt{\mu_{\mathbb{M}_{i}}^{+}\left(u_{i}\right) \mu_{\xi^{-}}^{+}\left(u_{i}\right)}+2 \sqrt{\nu_{\mathbb{M}_{i}}^{-}\left(u_{i}\right) v_{\xi^{-}}^{-}\left(u_{i}\right)}\right. \\
& +2 \sqrt{{v_{\mathbb{M}_{i}}^{+}\left(u_{i}\right) v_{\xi^{-}}^{+}\left(u_{i}\right)}^{*}+\sqrt{\pi_{\mathbb{M}_{i}}^{-}\left(u_{i}\right) \pi_{\xi^{-}}^{-}\left(u_{i}\right)}+\sqrt{\pi_{\mathbb{M}_{i}}^{+}\left(u_{i}\right) \pi_{\xi^{-}}^{+}\left(u_{i}\right)}} \\
& \left.+\sqrt{\left(1-v_{\mathbb{M}_{i}}^{-}\left(u_{i}\right)\right)\left(1-v_{\xi^{-}}^{-}\left(u_{i}\right)\right)}+\sqrt{\left(1-v_{\mathbb{M}_{i}}^{+}\left(u_{i}\right)\right)\left(1-v_{\xi^{-}}^{+}\left(u_{i}\right)\right)}\right]
\end{aligned}
$$

To measure the separation between LCTS alternatives, we adopt the IVIF-SMs given in Eqs. (36 and 37) to determine the weighted $\operatorname{SM~} \operatorname{Sim}_{3 w}\left(M_{i}, \xi^{+}\right)$to the alternatives $M_{i}$ and the IS $\xi^{+}$and the weighted SM $\operatorname{Sim}_{3 w}\left(M_{i}, \xi^{-}\right)$to the alternatives $M_{i}$ and the AIS $\xi^{-}$depicted in Table 12 .

Step 6: Assessment of relative closeness index (CI).

The relative CI of each option regarding IVIF-ISs is computed by

$$
C_{c}\left(M_{i}\right)=\frac{\operatorname{Sim}_{3 w}\left(M_{i}, \xi^{+}\right)}{\operatorname{Sim}_{3 w}\left(M_{i}, \xi^{+}\right)+\operatorname{Sim}_{3 w}\left(M_{i}, \xi^{-}\right)}, \quad i=1(1) m .
$$

Using Eq. (38), the relative CI $C_{c}\left(M_{i}\right)$ of each LCTS option is calculated and given in Table 12.

Step 7: Select the highest value, denoted by $C_{c}\left(M_{k}\right)$ to the values $C_{c}\left(M_{i}\right), i=1,2, \ldots, m$. And hence $M_{k}$ is the best alternative.

According to relative CC values (see Table 11), $M_{7}$ is obtained as the best LCTS option since it has the highest relative CC value (0.9780), while $M_{3}$ is the last one.

The result achieved by the developed approach was also compared to the different current decision-making approaches. IVIF-MABAC (Mishra et al., 2020a; Xue et al., 2016) is one of the simplest and most conventional MCDM methods, which is generally implemented as a standard tool for comparing the results obtained from different MCDM methods. A further MDCM approach, TOPSIS (Bai, 2013) describes the idea for choosing the option with minimum discrimination to IS and the maximum discrimination from the AIS, while generalized Choquet integral Yildiz and Ergul (2015) represents measurable evidence in intervals form, while fuzzy measures are real numbers. As a result, all these methods can be implemented for LCTS selection. Initially, DEs can utilize these MCDM methods to evaluate LCTS options and then calculate and compare their ranking outcomes and take the final decision. The computations of criteria weight are concluded with proposed similarity measures, whereas the outcomes are evaluated by the proposed one, MABAC, TOPSIS, COPRAS (Mishra et al., 2020c) and ANP-GCI approaches on IVIFSs. Table 13 depicts that LCTSs $M_{11}, M_{8}$ and $M_{4}$ normally share the first rank. As a result, LCTS $M_{11}$ comes out as the optimal one as per these comparisons, while the option $M_{3}$ is last in all methods. The outcomes indicate almost same preferences in all approaches. The proposed method can be easily understood and straightforwardly implemented. The performance of the IVIF-ARAS method was better than that of the extant approaches in the framework of decision making for LCTS selection on IVIFSs.

Next, Fig. 3 specifies the ranks obtained by three approaches assign various LCTSs as the most desirable ones; a definite degree of deviation can be examined. Two of the four current approaches that were compared to recognize the LCTS $M_{11}$ to be optimal, while one of them classify $M_{11}$ as the second one, as depicted in Fig. 3. According to the results, the remaining preference order is different for LCTS selection, representing a clear benefit 
Table 12 Results of IVIFTOPSIS method for LCTS selection

\begin{tabular}{lllll}
\hline LCTSs & $\operatorname{Sim}\left(M_{i}, \xi^{+}\right)$ & $\operatorname{Sim}\left(M_{i}, \xi^{-}\right)$ & $C_{c}\left(M_{i}\right)$ & Ranking \\
\hline$M_{1}$ & 0.9169 & 0.9666 & 0.4868 & 13 \\
$M_{2}$ & 0.9108 & 0.9793 & 0.4819 & 14 \\
$M_{3}$ & 0.9071 & 0.9742 & 0.4822 & 15 \\
$M_{4}$ & 0.9690 & 0.9423 & 0.5070 & 6 \\
$M_{5}$ & 0.9701 & 0.9342 & 0.5094 & 4 \\
$M_{6}$ & 0.9539 & 0.9533 & 0.5002 & 12 \\
$M_{7}$ & 0.9780 & 0.9304 & 0.5125 & 2 \\
$M_{8}$ & 0.9764 & 0.9249 & 0.5135 & 1 \\
$M_{9}$ & 0.9662 & 0.9317 & 0.5091 & 5 \\
$M_{10}$ & 0.9750 & 0.9355 & 0.5103 & 3 \\
$M_{11}$ & 0.9540 & 0.9399 & 0.5037 & 9 \\
$M_{12}$ & 0.9578 & 0.9563 & 0.5004 & 11 \\
$M_{13}$ & 0.9553 & 0.9515 & 0.5010 & 10 \\
$M_{14}$ & 0.9517 & 0.9300 & 0.5058 & 7 \\
$M_{15}$ & 0.9647 & 0.9473 & 0.5046 & 8 \\
\hline
\end{tabular}

by its useful and efficient evaluation process. Also, if the number of LCTS alternatives increases, the advantage will become more apparent. The outcomes of the new method introduced in this paper and those of the existing methods are depicted in Table 13. From Table 13, the correlation coefficients are greater than 0.8 except IVIF-TOPSIS (Bai, 2013). Also, the WS coefficients are greater than 0.808 except IVIF-TOPSIS method. The properties of the WS coefficient (Sałabun \& Urbaniak, 2020) specify that it is a suitable procedure for comparing the similarity of priorities, which means the similarity of preference order of LCTS option is high. As a result, it can be said that there is a highly strong relationship between ranking results. Thus, it can be concluded that the outcome of the developed approach has consistency with the extant approaches. When compared to currently employed procedures, IVIF-ARAS has the following advantages:

(a) The IVIF-ARAS works on the basis of a broader standard of ARAS with SMs to select the strategies to implement of LCTSs toward the achievements of sustainable development problems in comparison with IVIF-MABAC, IVIF-TOPSIS (Compromise programming), ANP-GCI and IVIF-COPRAS (Mishra et al., 2020c) methods because IVIF-ARAS model considers the score values (deviations) from optimal option while the extant models only consider a single attribute of the minimum discrimination from IVIF-IS and maximum discrimination from IVIF-AIS.

(b) The proposed model only evaluates IVIF-IS, whereas IVIF-TOPSIS needs to obtain both IVIF-IS and IVIF-AIS, and IVIF-WASPAS model (Mishra \& Rani, 2018b) utilizes IVIFWAO and IVIFWGO and IVIF-COPRAS (Mishra et al., 2020c) utilizes IVIFWAO to obtain the final outcomes. To conclude with, it can be said that for MCDM methods with more attributes or options, IVIF-ARAS is capable of to some extent increasing the operational effectiveness with a higher operability. 
Table 13 Comparison of ranking results of IVIF-ARAS method with extant methods

\begin{tabular}{lllll}
\hline LCTSs & $\begin{array}{l}\text { IVIF-TOPSIS } \\
\text { (Bai, 2013) }\end{array}$ & $\begin{array}{l}\text { GCI method Yildiz } \\
\text { and Ergul (2015) }\end{array}$ & $\begin{array}{l}\text { IVIF-MABAC } \\
\text { (Xue et al., 2016) }\end{array}$ & $\begin{array}{l}\text { Proposed } \\
\text { IVIF-ARAS } \\
\text { method }\end{array}$ \\
\hline$M_{1}$ & 13 & 14 & 13 & 14 \\
$M_{2}$ & 14 & 11 & 10 & 9 \\
$M_{3}$ & 15 & 15 & 15 & 15 \\
$M_{4}$ & 6 & 2 & 1 & 2 \\
$M_{5}$ & 4 & 3 & 5 & 5 \\
$M_{6}$ & 12 & 9 & 7 & 10 \\
$M_{7}$ & 2 & 8 & 9 & 6 \\
$M_{8}$ & 1 & 13 & 12 & 12 \\
$M_{9}$ & 5 & 6 & 8 & 7 \\
$M_{10}$ & 3 & 4 & 4 & 3 \\
$M_{11}$ & 9 & 1 & 2 & 1 \\
$M_{12}$ & 11 & 10 & 11 & 11 \\
$M_{13}$ & 10 & 7 & 6 & 8 \\
$M_{14}$ & 7 & 5 & 3 & 4 \\
$M_{15}$ & 8 & 12 & 14 & 13 \\
Correlation coefficient (rs) & 0.482 & 0.964 & 0.946 & - \\
WS coefficient & 0.6054 & 0.9720 & 0.9211 & - \\
\hline
\end{tabular}

\section{Conclusions}

The selection of most appropriate LCTS is directly affected by numerous diverse attributes; therefore, this selection problem can be assumed as MCDM problem. In comparison with conventional crisp sets or FSs or IFSs, IVIFSs offer better opportunities for handling reallife problems. Therefore, the developed method is extended with IVIFSs to implement its advantages. In this paper, we first combine similarity measures and ARAS method under IVIFSs context. Then, developed approach is utilized to choose the desirable low-carbon tourism strategy among 15 available options. In this paper, firstly novel similarity measures have been developed for IVIFSs because proposed measures are verified to be the important issues to handle the uncertainty. Secondly, the ARAS method has introduced using similarity measure and concepts of IVIFSs, where the DEs and attribute weights are completely unknown. The proposed similarity measures have been applied to compute the attribute weights. Further, a case study of LCTS selection has been taken to exemplify the practicality and feasibility of the present IVIF-ARAS approach. An assessment index procedure for LCTS options is developed, which comprises three dimensions: social, economy and environmental of criteria. These criteria involve of four, five and three sub-criteria, respectively, which are broadly assumed based on the literatures, research reports and DEs' knowledge in different regions. In addition, comparison has been discussed to confirm the strength of the results obtained by the introduced approach. The key outcomes of the IVIFARAS method are (i) sound process that can be constructive for choosing most desirable one among others, (ii) it is latest and straightforward method distinguished by its effectiveness in IVIFSs background and (iii) it employs a procedure to obtain more realistic attribute weights that increases the permanence of developed method. By comparing with 


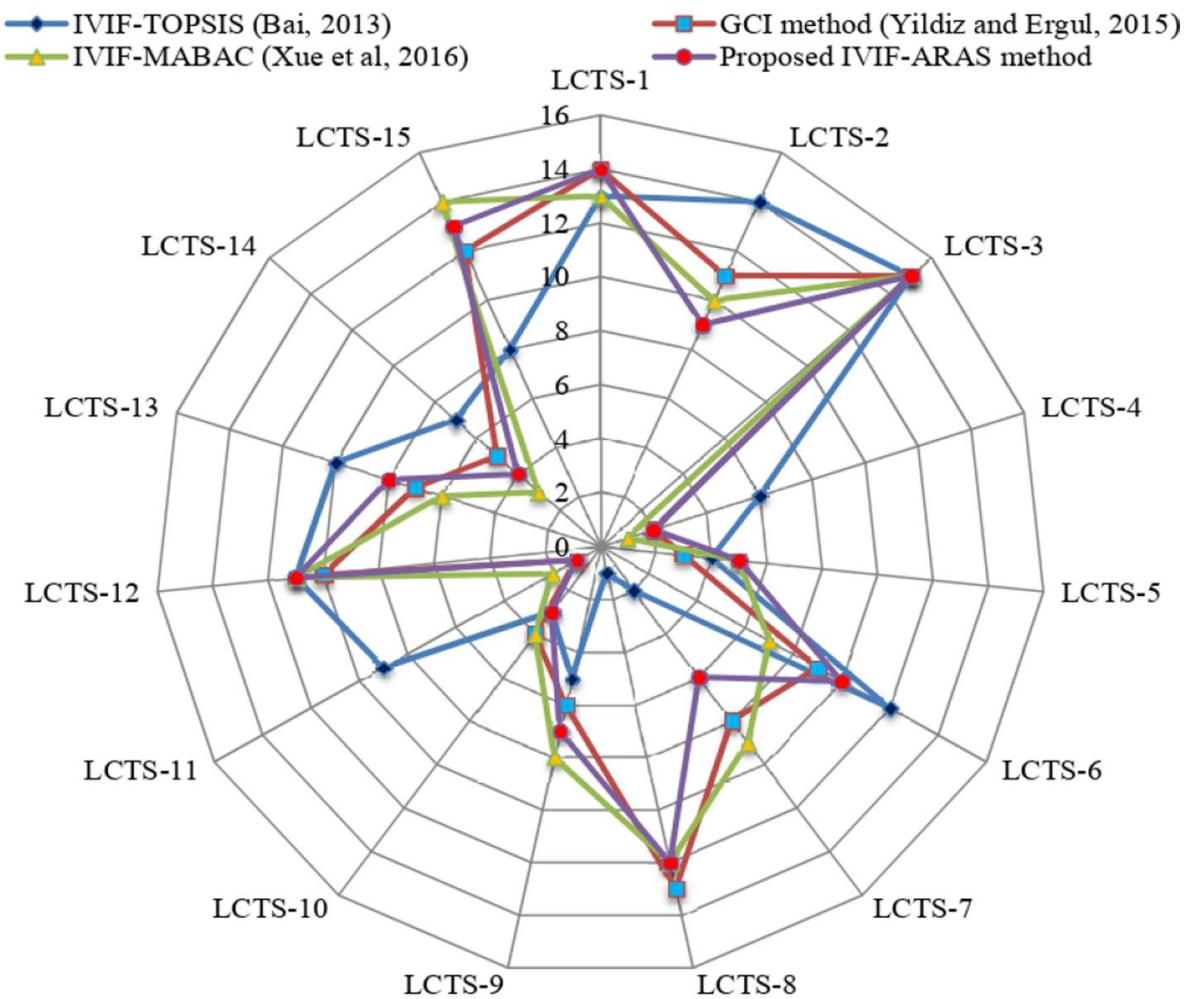

Fig. 3 LCTS options rankings for different MCDM methodologies

extant models, the IVIF-ARAS approach delivers a precise and proficient result of MCDM problems on IVIFSs setting.

Also, some potential limitations need to be considered in further study, which are: (i) this work miscarries to tackle with multifaceted LCTS assessment problem with interdependent criteria, (ii) more sustainability indicators can be considered in the assessment index structure, and (iii) further, the attitude of each expert is not calculated methodically.

In future studies, we will integrate the ARAS approach with different decision support methods, namely AHP, CRITIC, DEMATEL, SWARA, WASPAS and so on, and further consider the interaction among the criteria by using the Choquet integral. In addition, this research will be continued with anticipation that the method can be applied to other sustainable and green supplier selection problems, for example, sustainable supplier selection, medical waste treatment method selection and others.

\section{References}

Abdullah, L., Zulkifli, N., Liao, H., Herrera-Viedma, E., \& Al-Barakati, A. (2019). An interval-valued intuitionistic fuzzy Dematel method combined with Choquet integral for sustainable solid waste management. Engineering Applications of Artificial Intelligence, 82, 207-215. 
Akhavan, P., Barak, S., Maghsoudlou, H., \& Antucheviciene, J. (2015). FQSPM-SWOT for strategic alliance planning and partner selection; case study in a holding car manufacturer company. Technological and Economic Development of Economy, 21(2), 165-185.

Amirghodsi, S., Naeini, A.B., Makui, A., 2020. An Integrated Delphi-DEMATEL-ELECTRE method on gray numbers to rank technology providers. IEEE Transactions on Engineering Management, 1-17. https://doi.org/10.1109/TEM.2020.2980127.

Atanassov, K., \& Gargov, G. (1989). Interval valued intuitionistic fuzzy sets. Fuzzy Sets and Systems, 31(3), 343-349.

Bahrami, Y., Hassani, H., \& Maghsoudi, A. (2019). BWM-ARAS: A new hybrid MCDM method for Cu prospectivity mapping in the Abhar area, NW Iran. Spatial Statistics, 33, 100382.

Bai, Z.-Y. (2013). An interval-valued intuitionistic fuzzy Topsis method based on an improved score function. The Scientific World Journal, 2013, 6.

Balasubramaniam, P., \& Ananthi, V. P. (2014). Image fusion using intuitionistic fuzzy sets. Information Fusion, 20, 21-30.

Balezentis, T., \& Streimikiene, D. (2017). Multi-criteria ranking of energy generation scenarios with Monte Carlo simulation. Applied Energy, 185, 862-871.

Berger, L., Bréchet, T., Pestiaux, J., \& van Steenberghe, V. (2020). Case-study - The transition of Belgium towards a low carbon society: A macroeconomic analysis fed by a participative approach. Energy Strategy Reviews, 29, 100463.

Bonsu, N. O. (2020). Towards a circular and low-carbon economy: Insights from the transitioning to electric vehicles and net zero economy. Journal of Cleaner Production, 256, 120659.

Buyukozkan, G., \& Gocer, F. (2018). An extension of ARAS methodology under interval valued intuitionistic fuzzy environment for digital supply chain. Applied Soft Computing, 69, 634-654.

Büyüközkan, G., \& Gocer, F. (2017). An intuitionistic fuzzy MCDM approach for effective hazardous waste management. In C. Kahraman \& İU. Sari (Eds.), Intelligence systems in environmental management: Theory and applications (pp. 21-40). Springer International Publishing.

Büyüközkan, G., \& Göçer, F. (2017). Application of a new combined intuitionistic fuzzy MCDM approach based on axiomatic design methodology for the supplier selection problem. Applied Soft Computing, 52, 1222-1238.

Chatterjee, N., \& Bose, G. (2013). Selection of vendors for wind farm under fuzzy MCDM environment. International Journal of Industrial Engineering Computations, 4(4), 535-546.

Chen, Y., Liu, S., Wu, H., Zhang, X., \& Zhou, Q. (2020). How can Belt and Road countries contribute to glocal low-carbon development? Journal of Cleaner Production, 256, 120717.

Chen, L., Duan, D., Mishra, A. R., \& Alrasheedi, M. (2021). Sustainable third-party reverse logistics provider selection to promote circular economy using new uncertain interval-valued intuitionistic fuzzy-projection model. Journal of Enterprise Information Management. https://doi.org/10.1108/ JEIM-02-2021-0066

Cheng, Q., Su, B., \& Tan, J. (2013). Developing an evaluation index system for low-carbon tourist attractions in China - A case study examining the Xixi wetland. Tourism Management, 36, 314-320.

Cheng, X., Long, R., \& Chen, H. (2020). A policy utility dislocation model based on prospect theory: A case study of promoting policies with low-carbon lifestyle. Energy Policy, 137, 111134.

Chiesa, T., Gautam, A., 2009. Towards a low carbon travel \& tourism sector, World Economic Forum: Geneva, Switzerland.

Cho, Y.-J., Wang, Y., \& Hsu, L.L.-I. (2016). Constructing Taiwan's low-carbon tourism development suitability evaluation indicators. Asia Pacific Journal of Tourism Research, 21(6), 658-677.

Cojoianu, T. F., Clark, G. L., Hoepner, A. G. F., Veneri, P., \& Wójcik, D. (2020). Entrepreneurs for a low carbon world: How environmental knowledge and policy shape the creation and financing of green start-ups. Research Policy, 49(6), 103988.

Dadelo, S., Turskis, Z., Zavadskas, E. K., \& Dadeliene, R. (2012). Multiple criteria assessment of elite security personnel on the basis of ARAS and expert methods. Economic Computation and Economic Cybernetics Studies and Research, 46(4), 65-87.

Dahooie, J. H., Abadi, E. B. J., Vanaki, A. S., \& Firoozfar, H. R. (2018). Competency-based IT personnel selection using a hybrid SWARA and ARAS-G methodology. Human Factors and Ergonomics in Manufacturing \& Service Industries, 28(1), 5-16.

Dahooie, J. H., Firoozfar, H. R., \& Vanaki, A. S. (2019). An evaluation model of business intelligence for enterprise systems with interval-valued intuitive fuzzy ARAS. In T. Daim, M. Dabić, N. Başoğlu, J. R. Lavoie, \& B. J. Galli (Eds.), R\&D management in the knowledge era: Challenges of emerging technologies (pp. 261-282). Springer International Publishing.

Dengfeng, L., \& Chuntian, C. (2002). New similarity measures of intuitionistic fuzzy sets and application to pattern recognitions. Pattern Recognition Letters, 23(1), 221-225. 
Durbarry, R., \& Seetanah, B. (2015). The impact of long haul destinations on carbon emissions: The case of mauritius. Journal of Hospitality Marketing \& Management, 24(4), 401-410.

Dymova, L., \& Sevastjanov, P. (2016). The operations on interval-valued intuitionistic fuzzy values in the framework of Dempster-Shafer theory. Information Sciences, 360, 256-272.

Ecer, F. (2018). An integrated fuzzy AHP and ARAS model to evaluate mobile banking services. Technological and Economic Development of Economy, 24(2), 670-695.

Filimonau, V., Dickinson, J., Robbins, D., \& Huijbregts, M. A. J. (2011). Reviewing the carbon footprint analysis of hotels: Life cycle energy analysis (LCEA) as a holistic method for carbon impact appraisal of tourist accommodation. Journal of Cleaner Production, 19(17), 1917-1930.

Fu, Y.-K. (2019). An integrated approach to catering supplier selection using AHP-ARAS-MCGP methodology. Journal of Air Transport Management, 75, 164-169.

Ghenai, C., Albawab, M., \& Bettayeb, M. (2020). Sustainability indicators for renewable energy systems using multi-criteria decision-making model and extended SWARA/ARAS hybrid method. Renewable Energy, 146, 580-597.

Gössling, S., \& Buckley, R. (2016). Carbon labels in tourism: Persuasive communication? Journal of Cleaner Production, 111, 358-369.

Gössling, S., Hall, C.M., Weaver, D.B.J., (2012). Sustainable Tourism Futures. Routledge.

Gössling, S., Scott, D., \& Hall, C. M. (2015). Inter-market variability in CO2 emission-intensities in tourism: Implications for destination marketing and carbon management. Tourism Management, 46, 203-212.

Goswami, S., \& Mitra, S. (2020). Selecting the best mobile model by applying AHP-COPRAS and AHPARAS decision making methodology. International Journal of Data and Network Science, 4(1), 27-42.

Greco, S., Ishizaka, A., Tasiou, M., \& Torrisi, G. (2021). The ordinal input for cardinal output approach of non-compensatory composite indicators: The Promethee scoring method. European Journal of Operational Research, 288(1), 225-246.

Harris, S., Weinzettel, J., Bigano, A., \& Källmén, A. (2020). Low carbon cities in 2050? GHG emissions of European cities using production-based and consumption-based emission accounting methods. Journal of Cleaner Production, 248, 119206.

He, B., Cao, X., \& Gu, Z. (2020). Kinematics of underactuated robotics for product carbon footprint. Journal of Cleaner Production, 257, 120491.

He, J., Tu, X., (2020). On sustainable development of low-carbon tourism in Jiangxi Province. Journal of Physics: Conference Series. IOP Publishing, p. 022134.

He, J.B., Wang, Z., (2014). Research on low carbon transformation of tourism industry in China, Applied Mechanics and Materials. Trans Tech Publ, pp. 1470-1473.

He, J., Huang, Z., Mishra, A. R., \& Alrasheedi, M. (2021). Developing a new framework for conceptualizing the emerging sustainable community-based tourism using an extended interval-valued Pythagorean fuzzy SWARA-MULTIMOORA. Technological Forecasting and Social Change. https://doi.org/10.1016/j.techfore.2021.120955

Heidary Dahooie, J., Zavadskas, E. K., Vanaki, A. S., Firoozfar, H. R., Lari, M., \& Turskis, Z. (2019). A new evaluation model for corporate financial performance using integrated CCSD and FCMARAS approach. Economic Research-Ekonomska Istraživanja, 32(1), 1088-1113.

Horng, J.-S., Hu, M.-L.M., Teng, C.-C.C., \& Lin, L. (2014). Energy saving and carbon reduction behaviors in tourism - A perception study of asian visitors from a protection motivation theory perspective. Asia Pacific Journal of Tourism Research, 19(6), 721-735.

$\mathrm{Hu}, \mathrm{K} .$, \& Li, J. (2013). The entropy and similarity measure of interval valued intuitionistic fuzzy sets and their relationship. International Journal of Fuzzy Systems, 15(3), 279-288.

Huang, B., \& Mauerhofer, V. (2016). Low carbon technology assessment and planning-Case analysis of building sector in Chongming, Shanghai. Renewable Energy, 86, 324-331.

Intarapaiboon, P. (2014). New similarity measures for intuitionistic fuzzy sets. Applied Mathematical Sciences, 8(45), 2239-2250.

Iordache, M., Schitea, D., Deveci, M., Akyurt, İZ., \& Iordache, I. (2019). An integrated ARAS and interval type-2 hesitant fuzzy sets method for underground site selection: Seasonal hydrogen storage in salt caverns. Journal of Petroleum Science and Engineering, 175, 1088-1098.

Juvan, E., \& Dolnicar, S. (2014). Can tourists easily choose a low carbon footprint vacation? Journal of Sustainable Tourism, 22(2), 175-194.

Kersuliene, V., \& Turskis, Z. (2011). Integrated fuzzy multiple criteria decision making model for architect selection. Technological and Economic Development of Economy, 17(4), 645-666.

Kilic, H. S., Demirci, A. E., \& Delen, D. (2020). An integrated decision analysis methodology based on IF-DEMATEL and IF-ELECTRE for personnel selection. Decision Support Systems, 137, 113360. 
Kumar, A., Hussain, S.A.I., Rai, R.N., (2019). Optimization by AHP-ARAS of EDM process parameters on machining AA7050-10\%B4C composite. Springer Singapore, Singapore, pp. 285-296.

Lee, S.-H., Wu, S.-C., \& Li, A. (2018). Low-carbon tourism of small islands responding to climate change. World Leisure Journal, 60(3), 235-245.

Liao, C. N., Fu, Y. K., \& Wu, L. C. (2016). Integrated fuzzy AHP, ARAS-F AND MSGP methods for green supplier evaluation and selection. Technological and Economic Development of Economy, 22(5), 651-669.

Luo, Y., Jin, M., Ren, P., Liao, Z., Zhu, Z., (2014). Simulation and prediction of decarbonated development in tourist attractions associated with low-carbon economy. Sustainability, 6(4), 2320-2337. https://doi.org/10.3390/su6042320.

Makan, A., \& Fadili, A. (2020). Sustainability assessment of large-scale composting technologies using PROMETHEE method. Journal of Cleaner Production, 261, 121244.

McKercher, B., Prideaux, B., Cheung, C., \& Law, R. (2010). Achieving voluntary reductions in the carbon footprint of tourism and climate change. Journal of Sustainable Tourism, 18(3), 297-317.

McLennan, C.-1.J., Becken, S., Battye, R., So, K.K.F., . (2014). Voluntary carbon offsetting: Who does it? Tourism Management, 45, 194-198.

Meng, F., \& Chen, X. (2016). Entropy and similarity measure for Atannasov's interval-valued intuitionistic fuzzy sets and their application. Fuzzy Optimization and Decision Making, 15(1), 75-101.

Mishra, A. R. (2016). Intuitionistic fuzzy information measures with application in rating of township development. Iranian Journal of Fuzzy Systems, 13(3), 49-70.

Mishra, A. R., \& Rani, P. (2017). Information measures based Topsis method for multicriteria decision making problem in intuitionistic fuzzy environment. Iranian Journal of Fuzzy Systems, 14(6), 41-63.

Mishra, A. R., \& Rani, P. (2018a). Biparametric information measures-based TODIM technique for interval-valued intuitionistic fuzzy environment. Arabian Journal for Science and Engineering, 43(6), 3291-3309.

Mishra, A. R., \& Rani, P. (2018b). Interval-valued intuitionistic fuzzy WASPAS method: Application in reservoir flood control management policy. Group Decision and Negotiation, 27(6), 1047-1078.

Mishra, A. R., Rani, P., \& Pardasani, K. R. (2019). Multiple-criteria decision-making for service quality selection based on Shapley COPRAS method under hesitant fuzzy sets. Granular Computing, 4(3), 435-449.

Mishra, A. R., Rani, P., Pardasani, K. R., Mardani, A., Stević, Ž, \& Pamučar, D. (2020a). A novel entropy and divergence measures with multi-criteria service quality assessment using intervalvalued intuitionistic fuzzy TODIM method. Soft Computing, 24(15), 11641-11661.

Mishra, A. R., Rani, P., Krishankumar, R., Ravichandran, K. S., \& Kar, S. (2021a). An extended fuzzy decision-making framework using hesitant fuzzy sets for the drug selection to treat the mild symptoms of Coronavirus Disease 2019 (COVID-19). Applied Soft Computing, 103, 107155. https:// doi.org/10.1016/j.asoc.2021.107155

Mishra, A. R., Rani, P., \& Saha, A. (2021b). Single-valued neutrosophic similarity measure-based additive ratio assessment framework for optimal site selection of electric vehicle charging station. International Journal of Intelligent Systems. https://doi.org/10.1002/int.22523

Mishra, A. R., Chandel, A., \& Motwani, D. (2020b). Extended MABAC method based on divergence measures for multi-criteria assessment of programming language with interval-valued intuitionistic fuzzy sets. Granular Computing, 5, 97-117.

Mishra, A. R., Rani, P., Mardani, A., Pardasani, K. R., Govindan, K., \& Alrasheedi, M. (2020c). Healthcare evaluation in hazardous waste recycling using novel interval-valued intuitionistic fuzzy information based on complex proportional assessment method. Computers \& Industrial Engineering, 139, 106140. https://doi.org/10.1016/j.cie.2019.106140

Mishra, A. R., Singh, R. K., \& Motwani, D. (2020d). Intuitionistic fuzzy divergence measure based Electre method for performance of cellular mobile telephone service providers. Neural Computing and Applications, 32, 3901-3921. https://doi.org/10.1007/s00521-018-3716-6

Mu, Z., Jing, L., Xiaohong, Z., Lei, T., Xiao-na, F., Shan, C., (2011). Study on low-carbon economy model and method of Chinese tourism industry, Green Finance and sustainability: Environmentally-aware business models and technologies. IGI Global, pp. 492-525.

Narayanamoorthy, S., Geetha, S., Rakkiyappan, R., \& Joo, Y. H. (2019). Interval-valued intuitionistic hesitant fuzzy entropy based VIKOR method for industrial robots selection. Expert Systems with Applications, 121, 28-37.

Pekala, B., \& Balicki, K. (2017). Interval-valued intuitionistic fuzzy sets and similarity measure. Iranian Journal of Fuzzy Systems, 14(4), 87-98. 
Puška, A., Stojanović, I., \& Maksimović, A. (2019). Evaluation of sustainable rural tourism potential in Brcko district of Bosnia and Herzegovina using multi-criteria analysis. Operational Research in Engineering Sciences: Theory and Applications, 2(2), 40-54.

Radovic, D., Stevic, Z., Pamucar, D., Zavadskas, E.K., Badi, I., Antucheviciene, J., Turskis, Z., (2018). Measuring performance in transportation companies in developing countries: A novel rough ARAS model. Symmetry, 10(10), 434. https://doi.org/10.3390/sym10100434.

Rafieyan, E., Khorsand, R., \& Ramezanpour, M. (2020). An adaptive scheduling approach based on integrated best-worst and VIKOR for cloud computing. Computers \& Industrial Engineering, 140, 106272.

Rani, P., Jain, D., \& Hooda, D. (2018). Shapley function based interval-valued intuitionistic fuzzy VIKOR technique for correlative multi-criteria decision making problems. Iranian Journal of Fuzzy Systems, 15(1), 25-54.

Rani, P., Mishra, A. R., Mardani, A., Cavallaro, F., Alrasheedi, M., \& Alrashidi, A. (2020a). A novel approach to extended fuzzy TOPSIS based on new divergence measures for renewable energy sources selection. Journal of Cleaner Production, 257, 120352.

Rani, P., Mishra, A. R., \& Pardasani, K. R. (2020b). A novel WASPAS approach for multi-criteria physician selection problem with intuitionistic fuzzy type-2 sets. Soft Computing, 24(3), 2355-2367.

Rani, P., Mishra, A. R., Pardasani, K. R., Mardani, A., Liao, H., \& Streimikiene, D. (2019). A novel VIKOR approach based on entropy and divergence measures of Pythagorean fuzzy sets to evaluate renewable energy technologies in India. Journal of Cleaner Production, 238, 117936.

Rani, P., Mishra, A. R., Rezaei, G., Liao, H., \& Mardani, A. (2020c). Extended Pythagorean fuzzy Topsis method based on similarity measure for sustainable recycling partner selection. International Journal of Fuzzy Systems, 22(2), 735-747.

Rani, P., Mishra, A. R., Krishankumar, R., Ravichandran, K. S., \& Gandomi, A. H. (2020d). A new Pythagorean fuzzy based decision framework for assessing healthcare waste treatment. IEEE Transactions on Engineering Management. https://doi.org/10.1109/TEM.2020.3023707

Safaei Ghadikolaei, A., Khalili Esbouei, S., \& Antucheviciene, J. (2014). Applying fuzzy MCDM for financial performance evaluation of Iraninan companies. Technological and Economic Development of Economy, 20(2), 274-291.

Sahu, R., Dash, S. R., \& Das, S. (2021). Career selection of students using hybridized distance measure based on picture fuzzy set and rough set theory. Decision Making: Applications in Management and Engineering, 4(1), 104-126.

Sałabun, W., \& Urbaniak, K., (2020). A new coefficient of rankings similarity in decision-making problems. In International Conference on Computational Science (pp. 632-645). Cham: Springer. https://doi. org/10.1007/978-3-030-50417-5_4.

Shaharudin, M. S., Fernando, Y., Chiappetta Jabbour, C. J., Sroufe, R., \& Jasmi, M. F. A. (2019). Past, present, and future low carbon supply chain management: A content review using social network analysis. Journal of Cleaner Production, 218, 629-643.

Shariati, S., Yazdani-Chamzini, A., Salsani, A., \& Tamosaitiene, J. (2014). Proposing a new model for waste dump site selection: Case study of Ayerma phosphate mine. Inzinerine Ekonomika-Engineering Economics, 25(4), 410-419.

Shi, Y., Han, B., \& Zeng, Y. (2020). Simulating policy interventions in the interfirm diffusion of low-carbon technologies: An agent-based evolutionary game model. Journal of Cleaner Production, 250, 119449.

Song, Y., \& Wang, X. (2017). A new similarity measure between intuitionistic fuzzy sets and the positive definiteness of the similarity matrix. Pattern Analysis and Applications, 20(1), 215-226.

Song, Y., Wang, X., Quan, W., \& Huang, W. (2019). A new approach to construct similarity measure for intuitionistic fuzzy sets. Soft Computing, 23(6), 1985-1998.

Su, J.J.E.p., (2019). Impact of tourism resource development based on low-carbon mode: a case study of Guizhou ethnic areas. Ecological Processes, 8(1), 1-7. https://doi.org/10.1186/s13717-019-0176-6.

Teng, C.-C., Horng, J.-S., Hu, M.-L.M., \& Chen, P.-C. (2014). Exploring the energy and carbon literacy structure for hospitality and tourism practitioners: Evidence from hotel employees in Taiwan. Asia Pacific Journal of Tourism Research, 19(4), 451-468.

Thongdejsri, M., \& Nitivattananon, V. (2019). Assessing impacts of implementing low-carbon tourism program for sustainable tourism in a world heritage city. Tourism Review, 74(2), 216-234.

Turskis, Z., \& Zavadskas, E. K. (2010a). A new fuzzy additive ratio assessment method (ARAS-F). Case study: The analysis of fuzzy multiple criteria in order to select the logistic centers location. Transport, 25(4), 423-432.

Turskis, Z., \& Zavadskas, E. K. (2010b). A novel method for multiple criteria analysis: Grey additive ratio assessment (ARAS-G) method. Informatica, 21(4), 597-610.

UNWTO, (2008). Tourism: Responding to Global Challenges. 
Varmazyar, M., Dehghanbaghi, M., \& Afkhami, M. (2016). A novel hybrid MCDM model for performance evaluation of research and technology organizations based on BSC approach. Evaluation and Program Planning, 58, 125-140.

Wang, L., Wang, Y.-M., \& Martínez, L. (2020). Fuzzy TODIM method based on alpha-level sets. Expert Systems with Applications, 140, 112899.

Wei, C.-P., Wang, P., \& Zhang, Y.-Z. (2011). Entropy, similarity measure of interval-valued intuitionistic fuzzy sets and their applications. Information Sciences, 181(19), 4273-4286.

Wen, A., \& Jing, Y. (2011). EU Low-carbon revolution's influence on the Yangtze River Delta regional development and its countermeasures. Energy Procedia, 5, 2289-2302.

Wu, C., Luo, P., Li, Y., \& Ren, X. (2014). A new similarity measure of interval-valued intuitionistic fuzzy sets considering Its hesitancy degree and applications in expert systems. Mathematical Problems in Engineering, 2014, 16.

Xu, G.-L., Wan, S.-P., \& Xie, X.-L. (2015). A selection method based on MAGDM with interval-valued intuitionistic fuzzy sets. Mathematical Problems in Engineering, 2015, 13.

Xu, J., Yao, L., \& Mo, L. (2011). Simulation of low-carbon tourism in world natural and cultural heritage areas: An application to Shizhong District of Leshan City in China. Energy Policy, 39(7), 4298-4307.

Xu, Z.-S. (2007a). On similarity measures of interval-valued intuitionistic fuzzy sets and their application to pattern recognitions. Journal of Southeast University (english Edition), 23(1), 139-143.

$\mathrm{Xu}, \mathrm{Z}$. (2007b). Methods for aggregating interval-valued intuitionistic fuzzy information and their application to decision making. Control and Decision, 22(2), 215.

Xu, Z. S., \& Chen, J. (2008). An overview of distance and similarity measures of intuitionistic measures. International Journal of Uncertainty, Fuzziness and Knowledge-Based Systems, 16(04), 529-555.

Xue, Y.-X., You, J.-X., Lai, X.-D., \& Liu, H.-C. (2016). An interval-valued intuitionistic fuzzy MABAC approach for material selection with incomplete weight information. Applied Soft Computing, 38, 703-713.

Ye, J. (2012). Multicriteria decision-making method using the Dice similarity measure based on the reduct intuitionistic fuzzy sets of interval-valued intuitionistic fuzzy sets. Applied Mathematical Modelling, 36(9), 4466-4472.

Ye, J. (2013). Interval-valued intuitionistic fuzzy cosine similarity measures for multiple attribute decisionmaking. International Journal of General Systems, 42(8), 883-891.

Yeni, F. B., \& Özçelik, G. (2019). Interval-valued Atanassov intuitionistic Fuzzy CODAS method for multi criteria group decision making problems. Group Decision and Negotiation, 28(2), 433-452.

Yildiz, A., \& Ergul, E. U. (2015). A two-phased multi-criteria decision-making approach for selecting the best smartphone. South African Journal of Industrial Engineering, 26(3), 194-215.

Yu, O., \& Tsung-Lin, L. (2020). A study on local identity of low carbon tourism based on social exchange theory-a case study of Taiwan's characteristic hot spring area. International Journal of Organizational Innovation, 12(3), 355-366.

Zamani, M., Rabbani, A., Yazdani-Chamzini, A., \& Turskis, Z. (2014). An integrated model for extending brand based on fuzzy ARAS and ANP methods. Journal of Business Economics and Management, 15(3), 403-423.

Zavadskas, E. K., \& Turskis, Z. (2010). A new additive ratio assessment (ARAS) method in multicriteria decision-making. Ukio Technologinis Ir Ekonominis Vystymas, 16(2), 159-172.

Zavadskas, E. K., Turskis, Z., \& Bagocius, V. (2015). Multi-criteria selection of a deep-water port in the Eastern Baltic Sea. Applied Soft Computing, 26, 180-192.

Zeppel, H., \& Beaumont, N. (2013). Assessing motivations for carbon offsetting by environmentally certified tourism enterprises. Anatolia, 24(3), 297-318.

Zhang, C., Luo, L., Liao, H., Mardani, A., Streimikiene, D., \& Al-Barakati, A. (2020). A priority-based intuitionistic multiplicative UTASTAR method and its application in low-carbon tourism destination selection. Applied Soft Computing, 88, 106026.

Zhang, J. (2017). Evaluating regional low-carbon tourism strategies using the fuzzy Delphi- analytic network process approach. Journal of Cleaner Production, 141, 409-419.

Zhang, J., \& Zhang, Y. (2020). Low-carbon tourism system in an urban destination. Current Issues in Tourism, 23(13), 1688-1704.

Zhou, G., Singh, J., Wu, J., Sinha, R., Laurenti, R., \& Frostell, B. (2015). Evaluating low-carbon city initiatives from the DPSIR framework perspective. Habitat International, 50, 289-299.

Publisher's Note Springer Nature remains neutral with regard to jurisdictional claims in published maps and institutional affiliations. 